\title{
Confidence intervals with higher accuracy for short and long memory linear processes
}

\author{
Masoud M. Nasari and Mohamedou Ould-Haye \\ School of Mathematics and Statistics of Carleton University \\ Ottawa, ON, Canada
}

\begin{abstract}
In this paper an easy to implement method of stochastically weighing short and long memory linear processes is introduced. The method renders asymptotically exact size confidence intervals for the population mean which are significantly more accurate than their classical counterparts for each fixed sample size $n$. It is illustrated both theoretically and numerically that the randomization framework of this paper produces randomized (asymptotic) pivotal quantities, for the mean, which admit central limit theorems with smaller magnitudes of error as compared to those of their leading classical counterparts. An Edgeworth expansion result for randomly weighted linear processes whose innovations do not necessarily satisfy the Cramer condition, is also established.
\end{abstract}

\section{Introduction}

In a nonparametric framework, the central limit theorem (CLT) is a critical tool in drawing inference about the mean of a population. The CLT validates the use of the percentiles of the normal distribution to approximate those of the unknown sampling distribution of a centered partial sum of a given set of data. The next important step after establishing a CLT is to characterize the departure of the sampling distribution of the underlying partial sum functional from normality and to address the speed at which it vanishes. Expressed as a function of the sample size $n$, and without restricting the distribution of the data to the symmetrical ones, the error of the CLT is generally known to be of order $O(1 / \sqrt{n})$. Usually, the vanishing rate $1 / \sqrt{n}$ is referred to as the first order accuracy, correctness or efficiency of the CLT. The error of the CLT has been extensively studied by several authors, mainly using the Berry-Esseen inequality and the Edgeworth expansion. Both these methods show that in the i.i.d case, the CLT is usually first order efficient (cf., e.g., Bhattacharya and Rao [6], Senatov [30] and Shorack [31]). The same error rate of $1 / \sqrt{n}$ has also been shown to hold for some classes of weakly dependent data (cf., for example, Götze and Hipp [14], Lahiri [23]). 
There are a number of methods in the literature that deal with the problem of how to increase the accuracy of the CLT. The tilted approximation (cf. Barndorff-Nielson and Cox [4]) is a method of reducing the error admitted by the CLT. In this method, which is of theoretical interest, the densities of the partial sums of an i.i.d. data set are approximated by some tilting expressions. The error of this approximation is of order $O(1 / n)$. The drawback of the tilted approximation is that computing the tilting expressions requires the full knowledge of the cumulant generating function of the data which is usually unknown. Also, in the context of i.i.d. data, some other error reduction techniques are built around adjusting the cut-off points, the usual percentiles of standard normal distribution, by some additive correcting factors (cf., for example, Hall [17]). This method is called the Edgeworth correction and it requires estimating values of $r$-th moments of the data, where $r \leq k$, for some $k \geq 3$. The drawback of this method is that it tends to over-correct the coverage probability of the resulting confidence intervals, when the sample size is relatively small. This issue is the result of the significant deviation between the actual values of the moments of the population from their estimated values, when the sample is of small size. Another Edgeworth correction method was introduced in Eriksson [13]. The approach is essentially a randomization via adding a simulated set of values, from a specific class of distributions, to the original data. To specify the class of distributions from which the additive values are to be generated, it is assumed that the values of the second and third moments of the original data are known. Furthermore, Eriksson [13] provides only one example of the distributions which can be used to generate the additive random values from. The approach is interesting in general, however, it is unsuitable for use in a nonparametric framework. The bootstrap (cf., for example, Efron [11]) is another approach to reducing the error of the CLT that requires repeated re-sampling from a given set of data and, hence it can be intrinsically viewed as randomized jackknife. Despite its popularity and advantages, the bootstrap approach has some drawbacks. This approach relies on repeatedly re-sampling with replacement from a given data set which makes it computationally expensive. Furthermore, the use of the bootstrap in application poses the important question of how large the number of bootstrap replications, $B$, should be in order to achieve a higher accuracy for the CLT in contrast to its classical counterpart. While the general belief is that for achieving the goal of higher accuracy, ideally $B$ should be $\infty$ (cf. Yatracos [32]), some suggest that $B=500$ to $B=1000$ suffices (cf. Efron and Tibshirani [12]), others suggest that the higher accuracy is available uniformly in $B$. This means that the higher accuracy can still be achieved with replications as small as $B=9$ or $B=19$, for instance, (cf. Hall [16]). It seems that there are no results in the literature that would relate the required number of bootstrap replications, $B$, to the size of the sample at hand. Furthermore, when bootstrap is to be put in use for dependent data, via re-sampling with replacement, the i.i.d. nature of the bootstrap samples fails to preserve the covariance structure of the original data (cf. Csörgö et al. [10] for discussions on the effect of bootstrapping on the covariance structure of linear processes). Overcoming this issue requires some adjusted re-sampling schemes, such as over-lapping and non-overlapping block-bootstrapping techniques (cf., e.g., Lahiri [22] and Kim and 
Nordman [19], Götze and Künsch [15]), and the sieve and the augmented sieve (cf., e. g., Poskitt et al. [27]). When dealing with long memory data these various adapted for dependant data versions of the bootstrap suffer from a significant underestimation of the variance which in turn translates in producing confidence intervals with poor coverage probability even for samples with moderate sizes (cf. numerical studies in Section 5).

In this paper we introduce a method to stochastically weighing a given set of data in a multiplicative way that results in more accurate CLTs for linear processes. This approach creates a framework that requires neither re-sampling nor extra knowledge on the distribution of the data except for the same commonly assumed moment conditions for the classical CLT.

The idea of boosting the accuracy of the CLT via creating randomized versions of the (asymptotic) pivots of interest, that continue to possess the pivotal property for the parameter of interest, was first explored in Csörgö and Nasari [9] for i.i.d. data and in Csörgö et al. [10] for linear processes. The viewpoint in the latter papers is to create pivots that admit CLTs with smaller magnitude of error by replacing a data set by a randomly weighted version of it, where the multiplicative weights are of the form of functionals of symmetric multinomial random variables. A broader randomization framework for i.i.d. data was introduced in Nasari [25], which is flexible in the sense that it allows choosing the randomizing weights from a virtually unlimited class of random weights, including multinomial weights, with a window parameter to regulate the trade-off between the accuracy of the CLT and the volume of the resulting randomized confidence regions for the mean of the original data. The present paper constitutes a generalization of the randomization approach introduced in Nasari [25] for i.i.d. data to the case when the data form a short or long memory linear process.

As will be seen in this paper, introducing a controlled extra source of randomness in conjunction with the random mechanism that produces the original data can enhance the accuracy of the CLT. The refinement in the accuracy results from multiplying the original data by appropriately chosen random factors which do not change the nature of the given data set. More precisely, the multiplicative stochastic weights used in this paper are so that, if the original data set is of short memory then so is their randomized version. The same continues to hold true for randomized long memory linear processes. Moreover, in the case of long memory linear processes, the randomized pivots introduced in this paper tend to yield significantly better probabilities of coverage as compared to a number of the existing bootstrap methods including the sieve and the augmented sieve (cf. Section 5).

We conclude this section by an outline of the topics addressed in the rest of the paper. In Section 2 we introduce a class of randomized pivotal quantities for the value of the common mean of the population from which a linear process structured data set of size $n \geq 1$ is drawn. In Section 3 we derive a general Edgeworth expansion result for a class of randomly weighted linear processes which, in the univariate case, is an extension of the Edgeworth expansion obtained in Götze and Hipp [14] for sums of weakly dependent random vectors. This result, that is also of independent interest, is then used to illustrate the error reduction effect of the randomization framework in Section 2 on the 
CLT of the randomized pivots introduced in it. Section 4 is devoted to establishing the asymptotic validity of the randomized pivots introduced in Section 2 via deriving a CLT for them under more relaxed conditions than those required for the Edgeworth expansion result in Section 3. Section 4 also contains some discussions on the resulting randomized confidence intervals and some numerical demonstrations of their improved performance over that of their classical non-randomized counterparts. A numerical comparison of the performances of some methods of bootstrap and the randomization scheme introduced in this paper is presented in Section 5 for long memory data. Further results on Studentization of the randomized pivotal quantities are given in Section 6. Section 7 includes the proofs.

\section{Skewness reduction}

In this section we discuss the shortcoming of the leading measures of skewness which are defined primarily for i.i.d. data, when dealing with manipulations (functionals) of dependent data. Then, we consider a generalization of the criterion of skewness so that it is suitable for both stationary and i.i.d. data. This generalized definition of skewness is then used to introduce our approach to increase the accuracy of the CLT for linear processes.

When the sampling distribution of the normalized partial sums of a set of $n, n \geq$ 1, univariate i.i.d. random variables $Y_{1}, \ldots, Y_{n}$, admits an Edgeworth expansion, the Fisher-Pearson's measure of skewness $\mathbb{E}\left(Y_{1}-\mathbb{E}\left(Y_{1}\right)\right)^{3} /\left(\operatorname{Var}\left(Y_{1}\right)\right)^{3 / 2}$ becomes the coefficient of an expression of order $O(1 / \sqrt{n})$, as $n \rightarrow \infty$. In other words, the measure of skewness is the coefficient of the slowest vanishing term of the error admitted by the CLT. This property motivates viewing the skewness as the most important characteristic of the distribution of the data in measuring the departure from normality. The closer the value of the skewness is to zero, the more symmetrical the sampling distribution of the underlying partial sum of a given data set will be. The commonly used measures of skewness, such as the aforementioned Fisher-Pearson, are defined for the marginal distribution of an i.i.d. set of data with a finite third moment. This definition is suitable only for the i.i.d. case, as it does not account for the dependence when a stationary and dependent data of size $n$ form the summands of a partial sum which admits the CLT. The latter observation calls for a broader definition for skewness that is to take the partial sum of a set of random variables into consideration. This means that skewness should naturally be defined for the sampling distribution of an underlying partial sum functional rather than for the marginal distribution of its summands. The need for such a definition for skewness in the context of this paper stemmed from our need for a proper measure of skewness when studying the problem of boosting the accuracy of pivotal quantities of the form of partial sums for the mean of linear processes. Prior to defining an appropriate measure of skewness for stationary linear processes, we first set up the definition of linear processes of consideration. To formally state our results in this section, and also for the use throughout this paper, we let $X_{1}, \ldots, X_{n}$ be the first 
$n \geq 1$ terms of the linear process $\left\{X_{t}: t \geq 1\right\}$ defined as

$$
X_{t}=\mu+\sum_{k=0}^{\infty} a_{k} \zeta_{t-k}, t \geq 1
$$

where $\mu, \mu \in \mathbb{R}$, is the mean of the process, $\left\{a_{k} ; k \in \mathbb{Z}\right\}$ is a sequence of real numbers such that $\sum_{k=0}^{\infty} a_{k}^{2}<\infty$ and $\left\{\zeta_{k}: k \in \mathbb{Z}\right\}$ are i.i.d. white noise innovations with $\mathbb{E} \zeta_{1}=0$ and $0<\sigma^{2}:=\operatorname{Var}\left(\zeta_{1}\right)<\infty$. Moreover, we assume that $X_{t}$ are non-degenerate with a finite variance $\gamma_{0}:=\mathbb{E} X_{1}^{2}-\mu^{2}:=\sigma^{2} \sum_{k=0}^{\infty} a_{k}^{2}-\mu^{2}$ and the autocovariance function

$$
\gamma_{h}:=\operatorname{Cov}\left(X_{s}, X_{s+h}\right)=\mathbb{E}\left[\left(X_{s}-\mu\right)\left(X_{s+h}-\mu\right)\right], h \geq 0, s \geq 1 .
$$

In this paper we consider two types of the linear process (2.1). $(i)$ when $\sum_{k=0}^{\infty}\left|a_{k}\right|=\infty$. In this case we refer to $X_{t}$ as a long memory linear process. In particular, we consider the case when, as $k \rightarrow \infty$, for some $c>0$ we have $a_{k} \sim c k^{d-1}$, where $0<d<1 / 2$. We refer to $d$ as the memory parameter. The other type of linear processes considered in this paper is $(i i)$ when $\sum_{k=0}^{\infty}\left|a_{k}\right|<\infty$. In this case we refer to $X_{t}$ as a short memory linear process. To unify our notation we define the memory parameter $d$ for short memory linear processes as $d=0$.

Consider the classical pivotal quantity for $\mu, T_{n, X}: \mathbb{R}^{n+1} \rightarrow \mathbb{R}$, that is a normalized partial sum functional, defined as

$$
T_{n, X}=\sum_{i=1}^{n}\left(X_{i}-\mu\right) /\left(\operatorname{Var}\left(\sum_{i=1}^{n} X_{i}\right)\right)^{1 / 2}
$$

When $\mathbb{E}\left|X_{1}\right|^{3}<\infty$, we define the skewness measure of the pivotal quantity $T_{n, X}$ as follows:

$$
\beta_{T_{n, X}}:=\mathbb{E}\left(T_{n, X}\right)^{3}=\mathbb{E}\left(\sum_{i=1}^{n}\left(X_{i}-\mu\right)\right)^{3} /\left(\operatorname{Var}\left(\sum_{i=1}^{n} X_{i}\right)\right)^{3 / 2} .
$$

In view of the stationarity of $X_{t}$, and without loss of generality, we assume that $\mu=0$ and expand the skewness measure $\beta_{T_{n, X}}$ as follows:

$$
\begin{aligned}
\beta_{T_{n, X}} & =\mathbb{E}\left(\sum_{i=1}^{n} X_{i}\right)^{3} /\left(\operatorname{Var}\left(\sum_{i=1}^{n} X_{i}\right)\right)^{3 / 2} \\
& =\mathbb{E}\left(X_{1}^{3}\right) /\left(\sqrt{n}\left(\gamma_{0}+2 \sum_{h=1}^{n}\left(1-\frac{h}{n}\right) \gamma_{h}\right)^{3 / 2}\right) \\
& +3\left(\sum_{h=1}^{n}\left(1-\frac{h}{n}\right)\left(\mathbb{E}\left(X_{1}^{2} X_{1+h}\right)+\mathbb{E}\left(X_{1} X_{1+h}^{2}\right)\right)\right) /\left(\sqrt{n}\left(\gamma_{0}+2 \sum_{h=1}^{n}\left(1-\frac{h}{n}\right) \gamma_{h}\right)^{3 / 2}\right) \\
& +6\left(\sum_{h=1}^{n-1} \sum_{h^{\prime}=1}^{n-h-1}\left(1-\frac{h+h^{\prime}}{n}\right) \mathbb{E}\left(X_{1} X_{1+h} X_{1+h+h^{\prime}}\right)\right) /\left(\sqrt{n}\left(\gamma_{0}+2 \sum_{h=1}^{n}\left(1-\frac{h}{n}\right) \gamma_{h}\right)^{3 / 2}\right) .
\end{aligned}
$$


By virtue of the preceding representation of the skewness of $T_{n, X}$, one can readily see that $\beta_{T_{n, X}} \rightarrow 0$, as $n \rightarrow \infty$. This is in agreement with the fact that, under appropriate conditions, $T_{n, X}$ has a normal limiting distribution.

We now introduce a randomization approach to construct more symmetrical, and hence more accurate (cf. Theorem 3.1 ), versions of $T_{n, X}$, based on the data set $X_{1}, \ldots, X_{n}$, as in (2.1), as follows:

$$
T_{n, X, w}\left(\theta_{n}\right)=\left(\sum_{i=1}^{n}\left(w_{i}-\theta_{n}\right)\left(X_{i}-\mu\right)\right) / \sqrt{n \mathfrak{D}_{n, X, w}}
$$

where,

$$
\begin{aligned}
\mathfrak{D}_{n, X, w} & :=\mathbb{E}\left(w_{1}-\theta_{n}\right)^{2} \gamma_{0}+2 \mathbb{E}\left(\left(w_{1}-\theta_{n}\right)\left(w_{2}-\theta_{n}\right)\right) \sum_{h=1}^{n}\left(1-\frac{h}{n}\right) \gamma_{h} \\
& =\operatorname{Var}\left(\sum_{i=1}^{n}\left(w_{i}-\theta_{n}\right) X_{i}\right) / n
\end{aligned}
$$

The real valued constant $\theta_{n}$, to which we shall refer as the window constant, and the random weights used in the definition of the randomized pivot $T_{n, X, w}\left(\theta_{n}\right)$, as in (2.6), are to be determined in view of the following scenario, to which we shall refer as the scheme (RS).

\subsection{The Randomization Scheme}

(RS) Let $w_{1}, \ldots, w_{n}$ be either nonsymmetric and i.i.d. random variables with $\mathbb{E}\left|w_{1}\right|^{3}<\infty$ or have a symmetric multinomial distribution, i.e., Multinomial $(n ; 1 / n, \ldots, 1 / n)$. Furthermore, we assume $w_{i}$ are independent from the data $X_{i}, 1 \leq i \leq n$. Define

$$
\mathcal{H}_{n, X, w}(\theta):=\frac{\mathbb{E}\left(\sum_{i=1}^{n}\left(w_{i}-\theta\right) X_{i}\right)^{3}}{n} .
$$

Choose the window constant $\theta_{n}$, such that $\mathcal{H}_{n, X, w}\left(\theta_{n}\right)=0$ or $\mathcal{H}_{n, X, w}\left(\theta_{n}\right)=\delta_{n}$, where $\delta_{n} \rightarrow 0$, as $n \rightarrow \infty$.

The following relation (2.8) explains how the randomization scheme (RS) results in more symmetrical pivotal quantities $T_{n, X, w}\left(\theta_{n}\right)$, as in (2.6), as compared to their nonrandomized counterpart $T_{n, X}$, as in (2.3). To do so, we compute the skewness of $T_{n, X, w}\left(\theta_{n}\right)$. In what follows, for simplicity and without loss of generality, we assume that $\mu=0$. 


$$
\begin{aligned}
\beta_{T_{n, X, w}}\left(\theta_{n}\right) & :=\mathbb{E}\left(\sum_{i=1}^{n}\left(w_{i}-\theta_{n}\right) X_{i}\right)^{3} /\left(\operatorname{Var}_{X, w}\left(\sum_{i=1}^{n}\left(w_{i}-\theta_{n}\right) X_{i}\right)\right)^{3 / 2} \\
& =\mathcal{H}_{n, X, w}\left(\theta_{n}\right) \frac{n}{\left(\operatorname{Var}_{X, w}\left(\sum_{i=1}^{n}\left(w_{i}-\theta_{n}\right) X_{i}\right)\right)^{3 / 2}}
\end{aligned}
$$

From the preceding equation, it is evident that choosing a window constant $\theta_{n}$ in accordance with the randomization scheme (RS), translates in values for $\beta_{T_{n, X, w}}\left(\theta_{n}\right)$ which are zero or negligible. In other words, when a window constant $\theta_{n}$ is chosen based on the scheme (RS), the randomized pivot $T_{n, X, w}\left(\theta_{n}\right)$, as in (2.6), will be more symmetrical than its classical counterpart $T_{n, X}$ as in (2.3). The effect of this symmetrization on the accuracy of the CLT for $T_{n, X, w}\left(\theta_{n}\right)$, as in (2.6) , is discussed in Section 3 .

It is important to note that in the context of the scheme (RS), the skewness of the partial sum of a linear process can be made arbitral small without changing its dependence structure. The change in the dependence structure happens when the window constant equals the common mean of the weights. This remark is formulated in the following Corollary 2.1,

Corollary 2.1. Consider the randomization scheme (RS).

(a) (Existence of solution) For large sample size $n$, the equation $\mathcal{H}_{n, X, w}(\theta)=0$ has at least one real valued solution $\theta_{n}$.

(b) (Dependence structure Preservation) Let $\theta_{n}$ be a real solution to $\mathcal{H}_{n, X, w}(\theta)=\delta_{n}$, where $\delta_{n} \rightarrow 0$. Then, we have $\lim _{n \rightarrow \infty} \theta_{n} \neq E\left(w_{1}\right)$.

Remark 2.1. From Corollary 2.1 it is implied that $\theta_{n}$ cannot be equal to $E\left(w_{1}\right)$ in the context of the scheme (RS). This results in two important and desirable properties. Firstly, it prevents change in the covariance structure of the data after being multiplied by the non-centered random wights. More precisely, in view of (RS), if the original data $X_{i}$ have a short memory structure then so is their randomized version $\left(w_{i}-\theta_{n}\right) X_{i}$ and the same is also true for randomized long memory data. Secondly, non-centered weights yield randomized confidence intervals, for the parameter of interest $\mu=\mathbb{E}\left(X_{1}\right)$, whose lengths vanish as the sample size increases to $\infty$ (we refer to Subsection 4.2 for further details in this regard).

\section{The effect of scheme (RS) on the error of the CLT for randomized linear processes}

We use the Edgeworth expansion for a class of linear processes to illustrate the refinement that results from our randomization approach (RS), as in Subsection 2.1, which is primarily designed to reduce $\beta_{T_{n}, X}$, the skewness of the classical pivot $T_{n, X}$, as in (2.5). The choice of the Edgeworth expansion is due to the fact that it provides an explicit and direct link between the skewness of a partial sum pivot and the error admitted by 
its CLT.

The following Theorem 3.1 gives an Edgeworth expansion for the sampling distribution of partial sums of the random variables $\left(w_{i}-\theta_{n}\right)\left(X_{i}-\mu\right), 1 \leq i \leq n$, where $X_{1}, \ldots, X_{n}$ are data from some short memory linear processes as in (2.1).

Theorem 3.1. Assume that the data $X_{1}, \ldots, X_{n}$ are as in (2.1). Let the weights $w_{1}, w_{2}, \cdots$, be i.i.d. random variables, that are independent from the data. Also, let $\left\{\theta_{n}\right\}$ be a converging sequence of constants such that, $\lim _{n \rightarrow \infty} \theta_{n} \neq \mathbb{E}\left(w_{1}\right)$. Furthermore, assume that

(i) either $\sum_{k=0}^{\infty} a_{k}\left(w_{k+1}-\theta_{n}\right) \neq 0$, a.s. for all $n \geq 1$, and $\limsup _{u \rightarrow \infty}\left|\mathbb{E}\left(e^{i u \zeta_{1}}\right)\right|<1$,

or

$$
X_{1} \neq 0 \text { a.s., and } \limsup _{u \rightarrow \infty}\left|\mathbb{E}\left(e^{i u w_{1}}\right)\right|<1,
$$

(ii) There exists $\ell>0$ such that $\left|a_{k}\right| \leq(1 / \ell) e^{-\ell k}, \quad$ for all $k=0,1, \ldots$,

(iii) $\mathbb{E}\left|\zeta_{1}\right|^{5}<\infty$

(iv) $\mathbb{E}\left|w_{1}\right|^{5}<\infty$.

Then, we have for all $x \in \mathbb{R}$,

$$
P\left(T_{n, X, w}\left(\theta_{n}\right) \leq x\right)-\Phi(x)=\beta_{T_{n, X, w}}\left(\theta_{n}\right) H(x) / \sqrt{n}+O(1 / n),
$$

where $T_{n, X, w}(\theta)$ and $\beta_{T_{n, X, w}}(\theta)$ are, respectively, as in (2.6) and (2.8), $\Phi$ is the distribution function of the standard normal distribution and $H$ is a polynomial of degree 3.

We note that the conditions $\limsup \left|\mathbb{E}\left(e^{i u w_{1}}\right)\right|<1$ and $(i v)$ of Theorem 3.1, pose no practical restriction on the choice of the random weights, as the class of random weights that satisfy these conditions is virtually unlimited.

In view of the randomization scheme (RS), as in Subsection 2.1, and the relation (3.1), the smaller the $\beta_{T_{n, X, w}}\left(\theta_{n}\right)$ is, the closer the error of the CLT of $T_{n, X, w}\left(\theta_{n}\right)$ will be to $O(1 / n)$. In the case the weights $w_{i}$ and the window constant $\theta_{n}$ are chosen in such a way that $\beta_{T_{n, X, w}}\left(\theta_{n}\right)=0$ then, in view of (3.1), the CLT for $T_{n, X, w}\left(\theta_{n}\right)$ will be second order accurate, i.e., of magnitude $O(1 / n)$. On the other hand, when the linear process $X_{t}$, as in (2.1), is so that its innovations satisfy the Cramer condition, i.e., $\limsup _{u \rightarrow \infty}\left|\mathbb{E}\left(e^{i u \zeta_{1}}\right)\right|<1$, and also it satisfies conditions $(i i)$ - (iii) of Theorem 3.1 as well as the condition

$$
\left(i^{\prime}\right) \sum_{k=0}^{\infty} a_{k} \neq 0
$$


then, Theorem (2.8) of Götze and Hipp [14] and its Corollary (2.9), in the univariate case, imply that, for all $x \in \mathbb{R}$, the sampling distribution of $T_{n, X}$, as in (2.3), admits the following Edgeworth expansion:

$$
P\left(T_{n, X} \leq x\right)-\Phi(x)=\beta_{T_{n, X}} H(x) / \sqrt{n}+O(1 / n),
$$

where $\beta_{T_{n, X}}$, as in (2.5), is the skewness of $T_{n, X}$.

To compare the Edgeworth expansion (3.1) to (3.2), we consider linear processes whose innovations $\zeta_{j}$ satisfy the Cramer condition and $\sum_{k=0}^{\infty} a_{k} \neq 0$. We also consider weights that are non-degenerate i.i.d. and continuous with a finite fifth moment. In this case the first part of condition $(i)$ in Theorem 3.1 holds true. Now under the conditions $(i i)$, (iii), and (iv), one can see that (3.1) yields a smaller error when $\beta_{T_{n, X, w}}\left(\theta_{n}\right)$ is small. In particular, when $\beta_{T_{n, X, w}}\left(\theta_{n}\right)=0$, then the randomized pivot $T_{n, X, w}\left(\theta_{n}\right)$ is accurate of the second order while the classical $T_{n, X}$ remains accurate only of the first order, i.e., of order $O(1 / \sqrt{n})$.

Remark 3.1. We note that in view of Theorem 3.1, a randomly weighted partial sum of a linear process can admit an Edgeworth expansion even when the non-randomized partial sum of the original data fails to do so. This is due to the fact that in order to have the expansion (3.1), the crucial Cramer condition for the innovations $\zeta_{j}$, which is assumed in Götze and Hipp [14], can now be shifted to the weights $w_{i}$. In other words, when the innovations of the linear process at hand fail to satisfy the Cramer condition, then the expansion (3.2) may no longer be valid. On the other hand, for the same linear process, using some appropriate non-centered randomizing weights, as specified in Theorem 3.1, yields a valid Edgeworth expansion as in (3.1) for the resulting normalized randomized partial sum $T_{n, X, w}\left(\theta_{n}\right)$, provided that the original linear process is so that $X_{1} \neq 0$ a.s.. This constitutes an achievement in significantly enlarging the class of linear processes for which Edgewrorth expansion holds.

Remark 3.2. Further to the advantage of randomization discussed in Remark 3.1, it is also worth noting that when constructed using some continuous random weights, so that they satisfy the conventional conditions (iii) and (iv) of Theorem [3.1, the randomized pivot $T_{n, X, w}(\theta)$ admits the Edgeworth expansion (3.1) for the linear processes (2.1) even when $\sum_{k=0}^{\infty} a_{k}=0$, provided that (ii) holds true. This is true, since, in the case that the random weights are continuous, the first part of condition (i) of Theorem 3.1 holds true even when $\sum_{k=0}^{\infty} a_{k}=0$. In contrast, the Edgewroth expansion (3.2) for the classical $T_{n, X}$, as in (2.3), is valid only for short memory linear processes (2.1) for which we have $\sum_{k=0}^{\infty} a_{k} \neq 0$, provided that the innovation satisfy the Cramer condition and, conditions (ii) and (iii) of Theorem 3.1 also hold true.

We conclude this section by noting that in the context of Theorem 3.1 and under its conditions $(i)$ and $(i i)$, when $\mathbb{E}\left|\zeta_{1}\right|^{4}<\infty$ and $\mathbb{E}\left|w_{1}\right|^{4}<\infty, T_{n, X, w}(\theta)$, as in (2.6)), for all $x \in \mathbb{R}$, admits the expansion

$$
P\left(T_{n, X, w}\left(\theta_{n}\right) \leq x\right)-\Phi(x)=\beta_{T_{n, X, w}}\left(\theta_{n}\right) H(x) / \sqrt{n}+o(1 / \sqrt{n}) .
$$


The preceding expansion is a counterpart of (3.1) on assuming the existence of a finite fourth moment for the innovations and the weights. The proof of the validity of (3.1) also results from the proof of Theorem 3.1 given in Section 7 .

The discussions following Theorem 3.1 on the error reduction effect of the scheme (RS), remain valid in view of (3.3) with $o(1 / \sqrt{n})$ instead of $O(1 / n)$. Moreover, the advantages of the randomization discussed in Remarks 3.1 and 3.2 , also remain valid when $T_{n, X, w}(\theta)$ admits the expansion (3.3).

\section{CLT for randomized linear processes}

In this section we establish a CLT for the randomized pivot $T_{n, X, w}\left(\theta_{n}\right)$, as in (2.6), under less stringent conditions than those assumed in Theorem [3.1. The CLT in this section is valid for the random weights and window constants as characterized in the randomization scheme (RS) in Subsection 2.1.

For the use in Theorems 4.1, 4.2 and 6.1, we let the notation $\left(\Omega_{w}, \mathcal{F}_{w}, P_{w}\right)$ stand for the probability space on which the triangular or non-triangular random weights are defined.

Theorem 4.1. Let $X_{1}, \ldots, X_{n}$ be the first $n$ terms of the linear process (2.1) and consider the randomized pivot $T_{n, X, w}\left(\theta_{n}\right)$, as in (2.6), with the weights $w_{1}, \ldots, w_{n}$ and the window constant $\theta_{n}$ be as specified in the scheme (RS). Then, as $n \rightarrow \infty$, we have for all $x \in \mathbb{R}$

$$
P\left(T_{n, X, w}\left(\theta_{n}\right) \leq x \mid w_{1}, \ldots, w_{n}\right) \longrightarrow \Phi(x) \quad \text { in probability }-P_{w}
$$

and, consequently,

$$
P\left(T_{n, X, w}\left(\theta_{n}\right) \leq x\right) \longrightarrow \Phi(x)
$$

\subsection{Studentization}

We introduce the following $G_{n, X, w}\left(\theta_{n}, d\right)$ as a Studentized version of $T_{n, X, w}\left(\theta_{n}\right)$ that is valid for short and long memory processes as specified in Theorem 4.2.

$$
G_{n, X, w}\left(\theta_{n}, d\right)=n^{-1 / 2-d}\left(\sum_{i=1}^{n}\left(w_{i}-\theta_{n}\right)\left(X_{i}-\mu\right)\right) / \sqrt{q^{-2 d} \mathcal{S}_{n, q, w}},
$$

where $q=O\left(n^{1 / 2}\right)$,

$$
\mathcal{S}_{n, q, w}=\mathbb{E}\left(w_{1}-\theta_{n}\right)^{2} \bar{\gamma}_{0}+\mathbb{E}\left(\left(w_{1}-\theta_{n}\right)\left(w_{2}-\theta_{n}\right)\right) \sum_{h=1}^{q} \bar{\gamma}_{h}\left(1-\frac{h}{q}\right)
$$

and $\bar{\gamma}_{s}:=\sum_{j=1}^{n-s}\left(X_{j}-\bar{X}_{n}\right)\left(X_{j+s}-\bar{X}_{n}\right) / n, 0 \leq s \leq n-1$, are the sample autocovariances. The Studentized pivot $G_{n, X, w}\left(\theta_{n}, d\right)$ can also be viewed as a randomized version of the Studentized pivotal quantity

$$
T_{n}^{s t u}(d):=n^{1 / 2-d}\left(\bar{X}_{n}-\mu\right) /\left(q^{-2 d}\left(\bar{\gamma}_{0}+2 \sum_{h=1}^{q} \bar{\gamma}_{h}(1-h / q)\right)\right)^{1 / 2} .
$$


Our Studentizing sequence $q^{-2 d} \mathcal{S}_{n, q, w}$ for $G_{n, X, w}\left(\theta_{n}, d\right)$ is a generalization of $q^{-2 d}\left(\bar{\gamma}_{0}+\right.$ $\left.2 \sum_{h=1}^{q} \bar{\gamma}_{h}(1-h / q)\right)$, the Studentzing sequence for the non-randomized $T_{n}^{s t u}(d)$. Introduced in Abadir et al. [2], the random sequence $q^{-2 d}\left(\bar{\gamma}_{0}+2 \sum_{h=1}^{q} \bar{\gamma}_{h}(1-h / q)\right)$ is an adaptation of Bartlett-kernel heteroscedasticity and autocorrelation consistent (HAC) estimator (see, for example, Andrews [3]) that allows for long memory. Although, in this paper attention is restricted to our HAC-type Studentizing sequences of the form $q^{-2 d} \mathcal{S}_{n, q, w}$, we remark that it is also desirable to use other existing robust sample based estimators such as Robinson's periodogram based memory and autocorrelation consistent (MAC) estimator (cf. Robinson 28), to construct Studentizing sequences for the randomized sum $n^{-1 / 2-d}\left(\sum_{i=1}^{n}\left(w_{i}-\theta_{n}\right)\left(X_{i}-\mu\right)\right)$.

In what follows the notation $G_{n, X, w}\left(\theta_{n}, \hat{d}\right)$ in the case of a long memory process will stand for $G_{n, X, w}\left(\theta_{n}, d\right)$, where the memory parameter $d$ is replaced by its sample based estimate $\hat{d}$ as specified in the following Theorem 4.2 which establishes the CLT for $G_{n, X, w}\left(\theta_{n}, d\right)$ and it reads as follows.

Theorem 4.2. Consider $X_{1}, \ldots, X_{n}$, the first $n$ terms of the linear process (2.1), and let the weights $w_{1}, \cdots, w_{n}$ and the window constants $\theta_{n}$ be as in Theorem 4.1 .

(A) If the linear process (2.1) is of short memory, i.e., $\sum_{k=0}^{\infty}\left|a_{k}\right|<\infty$, and $\mathbb{E} \zeta_{1}^{4}<\infty$, then, as $n, q \rightarrow \infty$ such that $q=O\left(n^{1 / 2}\right)$, we have, for all $x \in \mathbb{R}$,

$$
P\left(G_{n, X, w}\left(\theta_{n}, 0\right) \leq x \mid w_{1}, \ldots, w_{n}\right) \longrightarrow \Phi(x) \text { in probability }-P_{w},
$$

and, consequently,

$$
P\left(G_{n, X, w}\left(\theta_{n}, 0\right) \leq x\right) \longrightarrow \Phi(x), t \in \mathbb{R} .
$$

(B) Let the linear process (2.1) be of long memory such that $\mathbb{E} \zeta_{1}^{4}<\infty$ and, as $k \rightarrow \infty$, $a_{k} \sim c k^{d-1}$, for some $c>0$, where $0<d<1 / 2$. Then, as $n, q \rightarrow \infty$ such that $q=O\left(n^{1 / 2}\right)$, we have for all $x \in \mathbb{R}$,

$$
\begin{aligned}
& P\left(G_{n, X, w}\left(\theta_{n}, d\right) \leq x \mid w_{1}, \ldots, w_{n}\right) \longrightarrow \Phi(x) \text { in probability }-P_{w}, \\
& P\left(G_{n, X, w}\left(\theta_{n}, \hat{d}\right) \leq x \mid w_{1}, \ldots, w_{n}\right) \longrightarrow \Phi(x) \text { in probability }-P_{w}
\end{aligned}
$$

and, consequently,

$$
\begin{aligned}
& P\left(G_{n, X, w}\left(\theta_{n}, d\right) \leq x\right) \longrightarrow \Phi(x), x \in \mathbb{R}, \\
& P\left(G_{n, X, w}\left(\theta_{n}, \hat{d}\right) \leq x\right) \longrightarrow \Phi(x), x \in \mathbb{R},
\end{aligned}
$$

where $\hat{d}$ is an estimator of the memory parameter $d$ such that $\hat{d}-d=o_{P}(1 / \log n)$.

When the linear process in Theorem 4.2 is of long memory with memory parameter $d$, there are a number of estimators $\hat{d}$ in the literature that can be used to estimate $d$. The MLE of $d$, with the Haslett and Raftery [18] method used to approximate 
the likelihood and, Whittle estimator (cf. Künsch [21] and Robinson [29]) are two examples of the estimators of the memory parameter $d$. For more on estimators for the memory parameter and their asymptotic behavior, we refer to Bhansali and Kokoszka [5], Moulines and Soulier [24] and references therein.

\subsection{Randomized confidence intervals}

By virtue of Theorem 4.2, for the parameter of interest $\mu$, the mean of the linear process (2.1), the Studentized randomized pivotal quantity $G_{n, X, w}\left(\theta_{n}, d\right)$, as in (4.3), yields asymptotically exact size randomized confidence intervals with nominal size $100(1-\alpha) \%$, $0<\alpha<1$, which are of the form:

$$
I_{n, X, w}\left(\theta_{n}\right):=\left[\min \left\{\mathcal{A}_{n, X, w, \alpha}\left(\theta_{n}\right), \mathcal{B}_{n, X, w, \alpha}\left(\theta_{n}\right)\right\}, \max \left\{\mathcal{A}_{n, X, w, \alpha}\left(\theta_{n}\right), \mathcal{B}_{n, X, w, \alpha}\left(\theta_{n}\right)\right\}\right],
$$

where

$$
\begin{aligned}
& \mathcal{A}_{n, X, w, \alpha}\left(\theta_{n}\right)=\left(\sum_{i=1}^{n}\left(w_{i}-\theta_{n}\right) X_{i}-z_{\alpha / 2} n^{1 / 2+d} \sqrt{q^{-2 d} \mathcal{S}_{n, q, w}}\right) /\left(\sum_{i=1}^{n}\left(w_{i}-\theta_{n}\right)\right) \\
& \mathcal{B}_{n, X, w, \alpha}\left(\theta_{n}\right)=\left(\sum_{i=1}^{n}\left(w_{i}-\theta_{n}\right) X_{i}+z_{\alpha / 2} n^{1 / 2+d} \sqrt{q^{-2 d} \mathcal{S}_{n, q, w}}\right) /\left(\sum_{i=1}^{n}\left(w_{i}-\theta_{n}\right)\right)
\end{aligned}
$$

and $z_{\alpha / 2}$ is the $100(1-\alpha / 2)$-th percentile of the standard normal distribution. The length of the randomized confidence interval $I_{n, X, w}\left(\theta_{n}\right)$ is

$$
\operatorname{length}\left(I_{n, X, w}\left(\theta_{n}\right)\right)=2 z_{\alpha / 2} n^{1 / 2+d} \sqrt{q^{-2 d} \mathcal{S}_{n, q, w}} /\left|\sum_{i=1}^{n}\left(w_{i}-\theta_{n}\right)\right| .
$$

Remark 4.1. Along the lines of the proof of Theorem 4.2 in Section 7 it is shown that for $0 \leq d<1 / 2, q^{-2 d} \mathcal{S}_{n, q, w}$ asymptotically coincides with $\operatorname{Var}\left(n^{-1 / 2-d} \sum_{i=1}^{n}\left(w_{i}-\theta_{n}\right) X_{i}\right)$. In view of the latter asymptotic equivalence together with (4.6), one can see that when $\theta_{n}=\mathbb{E}\left(w_{1}\right)$, the length of the randomized confidence interval $I_{n, X, w}\left(\theta_{n}\right)$ fails to vanish as $n, q \rightarrow \infty$ such that $q=O\left(n^{1 / 2}\right)$. In view of Corollary 2.1. choosing $\theta_{n}$ to be equal to $\mathbb{E}\left(w_{1}\right)$ is not possible in the framework of the scheme (RS), as in Subsection 2.1 . Therefore, (RS) results in randomized confidence intervals whose limiting lengths is zero as $n, q \rightarrow \infty$ such that $q=O\left(n^{1 / 2}\right)$.

Tables 1 and 2 below provide an empirical comparison of the performance of the randomized confidence interval $I_{n, X, w}\left(\theta_{n}\right)$, as in (4.5), and that of

$$
I_{n, X}=\bar{X}_{n} \pm z_{\alpha / 2} n^{-1 / 2+d} \sqrt{q^{-2 d}\left(\bar{\gamma}_{0}+2 \sum_{h=1}^{q} \bar{\gamma}_{h}(1-h / q)\right)}
$$


which is constructed based on the classical $T_{n}^{s t u}(d)$, as in (4.4). The lag-length or the bandwidth $q$ was chosen based on relation (2.14) of Abadir et al. 2] in each case. More precisely, in Tables 1 and 2 we let $q$ be ceiling $\left(n^{1 / 3}\right)$ for the examined short memory linear process.

It is easy to see that the length of $I_{n, X}$ has the form:

$$
\text { length }\left(I_{n, X}\right)=2 z_{\alpha / 2} n^{-1 / 2+d} \sqrt{q^{-2 d}\left(\bar{\gamma}_{0}+2 \sum_{h=1}^{q} \bar{\gamma}_{h}(1-h / q)\right)} .
$$

The results in Tables 1 and 2 are at the nominal level of $95 \%$ with $\mathbf{z}_{\alpha / 2}=1.96$, and based on 2000 replications of the therein specified short memory AR(1) data, i.e., when $X_{t}=$ $\phi X_{t-1}+\zeta_{t}$, where $\zeta_{t}$ have standardized, heavily skewed, lognormal $(0,1)$ distribution. For the randomized confidence intervals in Tables 1 and 2, 2000 sets of therein specified random weights also generated simultaneously with the data. We note in passing that the processes considered in Tables 1 and 2 are of the form (2.1).

\begin{tabular}{||l|l||cc|}
\multicolumn{1}{c}{} & $\mathrm{I}_{n, X, w}\left(\theta_{n}\right)$ & $\mathrm{I}_{n, X}$ \\
$\mathrm{~N}$ & weights & Bernoulli $(1 / 4)$ & $\mathrm{NA}$ \\
& $\theta_{n}$ & $1 / 4+0.14$ & $\mathrm{NA}$ \\
& length & 1.81 & 1.43 \\
& coverage & 0.9155 & 0.8615 \\
\hline \hline \multirow{3}{*}{$\mathrm{n}=400$} & weights & Bernoulli $(1 / 4)$ & $\mathrm{NA}$ \\
& $\theta_{n}$ & $1 / 4+0.1$ & $\mathrm{NA}$ \\
& length & 1.68 & 1.11 \\
& coverage & 0.9365 & 0.8865 \\
\hline
\end{tabular}

Table 1: $\operatorname{AR}(1)$ with $\phi=0.8$

\begin{tabular}{|c|c|c|c|}
\hline & & $\mathrm{I}_{n, X, w}\left(\theta_{n}\right)$ & $\mathrm{I}_{n, X}$ \\
\hline \multirow{4}{*}{$\mathrm{n}=200$} & weights & Multinomial $(n ; 1 / n, \ldots, 1 / n)$ & NA \\
\hline & $\theta_{n}$ & $1-0.27$ & NA \\
\hline & length & 1.91 & 1.43 \\
\hline & coverage & 0.912 & 0.866 \\
\hline \multirow{4}{*}{$n=400$} & weights & Multinomial $(n ; 1 / n, \ldots, 1 / n)$ & NA \\
\hline & $\theta_{n}$ & $1+0.23$ & NA \\
\hline & length & 1.59 & 1.11 \\
\hline & coverage & 0.942 & 0.8995 \\
\hline
\end{tabular}

Table 2: $\operatorname{AR}(1)$ with $\phi=0.8$ 


\section{Comparison to bootstrap confidence intervals for long memory linear processes}

In the context of long memory data, the residual resampling method, known as the sieve bootstrap (cf., e. g., Poskitt [26]), is built around approximating a long memory process by approximating its infinite auto regressive representation by a truncated one. However, this approximation produces error terms which tend to be significant (cf. Table 3). The approximation significantly underestimates the variance of the statistic of interest, which is the sample mean in the context of this paper, based on long memory linear process structured data. The underestimation of the variance translates in poor coverage probabilities of the confidence intervals constructed using the (raw) sieve, as can be seen in Table 3. Filtered sieve introduced by Poskitt et al. [27] is a modification of the sieve that tends to improve upon the coverage probability of the sieve. It essentially consists of applying the raw sieve to a filtered series (basically to a truncated version of the infinite sum $(1-B)^{\hat{d}} X_{t}$, where $\hat{d}$ is an estimator of the long memory parameter $d$ ) and then unfilter the resulting bootstrapped error series $\hat{\epsilon}_{t}^{*}$ (again by considering the truncated sum $\left.(1-B)^{-\hat{d}_{\hat{\epsilon}}^{*}}\right)$.

The block bootstrap is another method of drawing a bootstrap sample from a given set of dependent and temporal observations with the goal of preventing the i.i.d. nature of the naive bootstrap from dismissing the dependence and chronological order of the data. The method essentially consists of first partitioning the data into a number of blocks and then ressampling from these blocks. This method was first introduced by Künsch [20] for short memory linear processes. The validity of this resampling scheme for both short and long memory linear processes has been investigated in a number of papers, e. g., Götze and Künsch [15] and Kim and Nordman [19].

Although, with a relatively large number of replications, the existing bootstrap methods tend to perform well for short memory data, it is not the case when they are applied to long memory data. In our numerical study below, we consider three methods of constructing bootstrap confidence intervals for the mean of a population from which long memory structured linear process data sets are simulated. In Table 3 we compare the performance of the resulting confidence intervals from the three bootstrap methods sieve, filtered or augmented sieve and the block bootstrap for long memory linear processes to those of the randomized confidence interval $I_{n, X, w}($.$) , as in (4.5), and the classical I_{n, X}$, as in (4.7), in terms of their respective empirical probabilities of coverage.

In Table 3, $I_{\text {AugSiv }}, I_{B l o c}$ and $I_{S i v}$ stand, respectively, for the augmented sieve, blockbootstrap and the sieve confidence intervals at nominal level of $95 \%$. Each of these bootstrap confidence intervals is constructed based on $B=1000$ bootstrap replications to estimate the cut-off points for each of the 2000 sets of simulated data. In Table 3, Mult is a short hand notation for the symmetric multinomial distribution, i.e., Multinomial $(n ; 1 / n, \cdots, 1 / n)$. For all examined confidence intervals, the simulated observations in Table 3, are generated from the fractionally integrated model $\mathrm{X}_{t}=(1-\mathrm{B})^{-\mathrm{d}} \zeta_{t}$, where, $\mathrm{B}$ is the back-shift operator, i.e., $\mathrm{B} \zeta_{t}=\zeta_{t-1}$, $\mathrm{d}$ is the memory 
parameter and $\zeta_{t}$ have standardized lognormal $(0,1)$ distribution. Similarly to Tables 1 and 2 , to construct the randomized confidence interval $I_{n, X, w}\left(\theta_{n}\right)$, for each simulated set of data, simultaneously, a set of random weights from symmetric multinomial distribution was generated. In Table 3 for the therein considered long memory linear process with $d=0.4$, we let $q$ be ceiling $\left(n^{1 / 2-d}\right)$.

\begin{tabular}{|c|c|c|c|c|c|c|}
\hline & & $\mathrm{I}_{n, X, w}\left(\theta_{n}\right)$ & $\mathrm{I}_{n, X}$ & $I_{\text {Bloc }}$ & $I_{\text {AugSiv }}$ & $I_{S i v}$ \\
\hline \multirow{4}{*}{$n=100$} & weights & Mult & NA & NA & NA & NA \\
\hline & $\theta_{n}$ & $1+0.97$ & NA & NA & NA & NA \\
\hline & length & 3.28 & 2.50 & 2.26 & 1.90 & 0.8695 \\
\hline & coverage & 0.935 & 0.8525 & 0.803 & 0.710 & 0.3795 \\
\hline \multirow{4}{*}{$n=200$} & weights & Mult & NA & NA & NA & NA \\
\hline & $\theta_{n}$ & $1+0.97$ & NA & NA & NA & NA \\
\hline & length & 3.20 & 2.45 & 2.25 & 1.83 & 0.8 \\
\hline & coverage & 0.9445 & 0.864 & 0.823 & 0.739 & 0.4035 \\
\hline
\end{tabular}

Table 3: Fractionally integrated long memory with $\mathrm{d}=0.4$

Remark 5.1. As it is evident from the numerical study presented in Table 3, the randomized confidence interval $I_{n, X, w}(\theta)$, introduced in this paper, as in (4.5), produces significantly more accurate confidence intervals as compared to the classical $I_{n, X}$, as in (4.7), and the three examined bootstrap confidence intervals. It is noteworthy that the higher accuracy of $I_{n, X, w}(\theta)$ can also be achieved using other random weights than the symmetric multinomial used in Table 3, provided that they are chosen according to the skewness reduction scheme (RS), as in Subsection 2.1.

\section{Complete Studentization}

In the Studentizing sequence $q^{-2 d} \mathcal{S}_{n, q, w}$ used in the definition of the Studentized randomized pivot $G_{n, X, w}\left(\theta_{n}, d\right)$, as defined in (4.3), we used the actual values of the moments of the wights, i.e., $\mathbb{E}\left(w_{1}\right), \mathbb{E}\left(w_{1}\right)^{2}$ and $\mathbb{E}\left(w_{1} w_{2}\right)$. The motive of the use of such a partial Studentization is justified considering that in the framework created by the scheme (RS), the distribution of the weights are usually known. Despite the validity of this reasoning, it is also desirable to investigate the asymptotic behavior of the completely Studentized version of $G_{n, X, w}\left(\theta_{n}, d\right)$. In the following Theorem 6.1 we establish a CLT result for the completely Studentized randomized pivotal quantity

$$
\hat{G}_{n, X, w}\left(\theta_{n}, d\right)=n^{-1 / 2-d}\left(\sum_{i=1}^{n}\left(w_{i}-\theta_{n}\right)\left(X_{i}-\mu\right)\right) / \sqrt{q^{-2 d} \hat{\mathcal{S}}_{n, q, w}},
$$


where $q=O\left(n^{1 / 2}\right)$,

$$
\hat{\mathcal{S}}_{n, q, w}=\frac{1}{n} \sum_{j=1}^{n}\left(w_{j}-\theta_{n}\right)^{2} \bar{\gamma}_{0}+2 q^{-1} \sum_{h=1}^{q} \bar{\gamma}_{h} \sum_{j=1}^{q-h}\left(w_{j}-\theta_{n}\right)\left(w_{j+h}-\theta_{n}\right),
$$

where $\bar{\gamma}_{s}, 0 \leq s \leq n-1$, are the sample autocovariances, as defined right after (4.3).

Theorem 6.1. Consider $X_{1}, \ldots, X_{n}$, the first $n$ terms of the linear process (2.1), and let the weights $w_{1}, \cdots, w_{n}$ and the window constants $\theta_{n}$ be as in Theorem 4.1.

(A) If the linear process (2.1) is of short memory, i.e., $\sum_{k=0}^{\infty}\left|a_{k}\right|<\infty$, and $\mathbb{E} \zeta_{1}^{4}<\infty$, then, as $n, q \rightarrow \infty$ such that $q=O\left(n^{1 / 2}\right)$, we have, for all $x \in \mathbb{R}$,

$$
P\left(\hat{G}_{n, X, w}\left(\theta_{n}, 0\right) \leq x \mid w_{1}, \ldots, w_{n}\right) \longrightarrow \Phi(x) \text { in probability }-P_{w},
$$

and, consequently,

$$
P\left(\hat{G}_{n, X, w}\left(\theta_{n}, 0\right) \leq x\right) \longrightarrow \Phi(x), t \in \mathbb{R} .
$$

(B) Let the linear process (2.1) be of long memory such that $\mathbb{E} \zeta_{1}^{4}<\infty$ and, as $k \rightarrow \infty$, $a_{k} \sim c k^{d-1}$, for some $c>0$, where $0<d<1 / 2$. Then, as $n, q \rightarrow \infty$ such that $q=O\left(n^{1 / 2}\right)$, we have for all $x \in \mathbb{R}$,

$$
\begin{aligned}
& P\left(\hat{G}_{n, X, w}\left(\theta_{n}, d\right) \leq x \mid w_{1}, \ldots, w_{n}\right) \longrightarrow \Phi(x) \text { in probability }-P_{w}, \\
& P\left(\hat{G}_{n, X, w}\left(\theta_{n}, \hat{d}\right) \leq x \mid w_{1}, \ldots, w_{n}\right) \longrightarrow \Phi(x) \text { in probability }-P_{w}
\end{aligned}
$$

and, consequently,

$$
\begin{aligned}
& P\left(\hat{G}_{n, X, w}\left(\theta_{n}, d\right) \leq x\right) \longrightarrow \Phi(x), x \in \mathbb{R}, \\
& P\left(\hat{G}_{n, X, w}\left(\theta_{n}, \hat{d}\right) \leq x\right) \longrightarrow \Phi(x), x \in \mathbb{R},
\end{aligned}
$$

where $\hat{d}$ is an estimator of the memory parameter $d$ such that $\hat{d}-d=o_{P}(1 / \log n)$.

The CLTs in the preceding Theorem 6.1 are counterparts of those in Theorem 4.2. 


\section{Proofs}

\section{Proof of Corollary 2.1}

The third degree polynomial, in $\theta, \mathcal{H}_{n, X, w}(\theta)$ can be written as:

$$
\begin{aligned}
\mathcal{H}_{n, X, w}(\theta) & =\frac{1}{n} \mathbb{E}\left(\sum_{i=1}^{n}\left(w_{i}-\theta\right) X_{i}\right)^{3} \\
& =-\frac{1}{n} \mathbb{E}\left(\sum_{i=1}^{n} X_{i}\right)^{3} \theta^{3} \\
& +\frac{3}{n} \mathbb{E}\left(\left(\sum_{i=1}^{n} w_{i} X_{i}\right)\left(\sum_{i=1}^{n} X_{i}\right)^{2}\right) \theta^{2}-\frac{3}{n} \mathbb{E}\left(\left(\sum_{i=1}^{n} w_{i} X_{i}\right)^{2}\left(\sum_{i=1}^{n} X_{i}\right)\right) \theta \\
& +\frac{1}{n} \mathbb{E}\left(\sum_{i=1}^{n} w_{i} X_{i}\right)^{3} .
\end{aligned}
$$

From the preceding representation, it can be seen that $\mathcal{H}_{n, X, w}(\theta)$ will have at least one real solution if for large $n$ we have

$$
\frac{1}{n} \mathbb{E}\left(\sum_{i=1}^{n} X_{i}\right)^{3} \neq 0 \text {. }
$$

In order to show that, as $n \rightarrow \infty$, (7.2) holds true, we first consider the case when the process $X_{t}$ is of short memory, i.e.

$$
X_{t}=\sum_{j=0}^{\infty} a_{j} \epsilon_{t-j}
$$

$\epsilon_{t}$ being i.i.d. white noise and

$$
\sum_{j=0}^{\infty}\left|a_{j}\right|<\infty \text { and } \sum_{j=0}^{\infty} a_{j} \neq 0 .
$$

In this case, on assuming that the innovations are nonsymmetric such that $\mathbb{E}\left(\epsilon_{1}^{3}\right) \neq 0$, it is easy to see that, as $n \rightarrow \infty$,

$$
\frac{1}{n} \mathbb{E}\left[\left(\sum_{i=1}^{n} X_{i}\right)^{3}\right] \rightarrow \mathbb{E}\left(\epsilon_{1}^{3}\right)\left(\sum_{j=0}^{\infty} a_{j}\right)^{3} \neq 0
$$

Now we consider the case when $X_{t}$ is of long memory, i.e.

$$
a_{i} \sim i^{(d-1)}, \quad \text { as } i \rightarrow \infty .
$$


Without loss of generality, we assume that $a_{i}>0$ for all $i$, as there could only be finitely many $i$ for which $a_{i} \leq 0$, and such $i$ 's will result in only a finite sum. Now, similarly to the formula (2.8), on assuming that $\mathbb{E}\left(\epsilon_{1}^{3}\right)>0$, as $n \rightarrow \infty$, we have

$$
\begin{aligned}
\frac{1}{n} \mathbb{E}[ & \left.\left(\sum_{i=1}^{n} X_{i}\right)^{3}\right]=\mathbb{E}\left(X_{1}^{3}\right)+3 \sum_{h=1}^{n}\left(1-\frac{h}{n}\right) \mathbb{E}\left(X_{1}^{2} X_{1+h}+X_{1} X_{1+h}^{2}\right) \\
& +\sum_{h=1}^{n-1} \sum_{h^{\prime}=1}^{n-h-1}\left(1-\frac{h+h^{\prime}}{n}\right) \mathbb{E}\left(X_{1} X_{1+h} X_{1+h+h^{\prime}}\right) \\
\geq & \sum_{h=1}^{n}\left(1-\frac{h}{n}\right) \mathbb{E}\left(X_{1}^{2} X_{1+h}\right)=\sum_{h=1}^{n}\left(1-\frac{h}{n}\right) \mathbb{E}\left(\epsilon_{1}^{3}\right) \sum_{i=0}^{\infty} a_{i}^{2} a_{i+h} \\
\geq & a_{0}^{2} \mathbb{E}\left(\epsilon_{1}^{3}\right) \sum_{h=1}^{n}\left(1-\frac{h}{n}\right) a_{h} \rightarrow \infty .
\end{aligned}
$$

In summary, we have proved that for both short and long memory, for $n$ large, (7.2) holds true. This completes the proof of part (a) of this corollary.

Now if we take the weights $w_{i}$ to be i.i.d. and nonsymmetric, with $\mathbb{E}\left(w_{i}-\mathbb{E}\left(w_{i}\right)\right)^{3} \neq 0$, we can see from (2.8) that

$$
\mathcal{H}_{n, X, w}\left(\mathbb{E}\left(w_{1}\right)\right)=\mathbb{E}\left(w_{i}-\mathbb{E}\left(w_{i}\right)\right)^{3} \mathbb{E}\left(X_{1}^{3}\right) \neq 0
$$

and hence $\mathbb{E}\left(w_{1}\right)$ cannot be a root of the polynomial $\mathcal{H}_{n, X, w}(\theta)$.

So, we conclude that there is a real root $\theta_{n}$ for $\mathcal{H}_{n, X, w}(\theta)$ and that this root is different from $\mathbb{E}\left(w_{1}\right)$. Choosing this root as a value for $\theta$ will guarantee a second order accuracy of the Theorem 3.1. Similar argument can be made when choosing the weights have a symmetric multinomial distribution. This is true since in that case, using the joint moment generating function of $\left(w_{1}, \ldots, w_{n}\right)$ and the fact that $\mathbb{E}\left(w_{i}\right)=1$, as $n \rightarrow \infty$, we have

$$
\mathcal{H}_{n, X, w}\left(\mathbb{E}\left(w_{1}\right)\right) \rightarrow \mathbb{E}\left(X_{1}^{3}\right) \neq 0
$$

and therefore for $n$ large, $\mathcal{H}_{n, X, w}\left(\mathbb{E}\left(w_{1}\right)\right) \neq 0$, which again guarantees that the real root we are seeking to achieve a negligible skewness, in the context of scheme (RS), cannot be equal to $\mathbb{E}\left(w_{1}\right)$. This completes the proof of part (b). Now the proof of the corollary is complete.

\section{Proof of Theorem 3.1}

We first define the following $\sigma$-fields to be used in the proof of this theorem.

$$
\begin{aligned}
\mathcal{D}_{j} & :=\sigma\left(w_{j-1}, \zeta_{j}\right), \quad j=0, \pm 1, \pm, 2, \ldots, \\
\mathcal{D}_{j, n} & :=\sigma\left(\zeta_{n}, w_{j-1}, \zeta_{j}\right), \quad j=0, \pm 1, \pm, 2, \ldots, \\
\mathcal{D}_{p}^{q} & :=\sigma\left\{\left(w_{j-1}, \zeta_{j}\right), \quad p \leq j \leq q\right\} .
\end{aligned}
$$


In order for the definition of $\mathcal{D}_{j}$ to hold true, we extended the weights $w_{1}, w_{2}, \ldots$, to

$$
\ldots, w_{-1}, w_{0}, w_{1}, \ldots
$$

Theorem 3.1 results from Theorem (2.8), and its Corollary (2.9), of Götze and Hipp [14]. More precisely, we show that under the conditions of Theorem 3.1, conditions (2.3) (2.6) of Götze and Hipp [14] hold true. In our case, (2.4) of Götze and Hipp [14] holds true trivially as $\mathcal{D}_{-\infty}^{n}$ is independent from $\mathcal{D}_{n+m}^{\infty}$, for all $m \geq 1$. To see why (2.3) holds, observe that

$$
\begin{aligned}
\mathbb{E}\left|\left(w_{n}-\theta_{n}\right)\left(X_{n}-\sum_{k=0}^{m} a_{k} \zeta_{n-k}\right)\right| & \leq \mathbb{E}\left|w_{1}-\theta_{n}\right| \mathbb{E}\left|X_{n}-\sum_{k=0}^{m} a_{k} \zeta_{n-k}\right| \\
& \leq \mathbb{E}\left|w_{1}-\theta_{n}\right| \mathbb{E}\left|\xi_{1}\right|(1 / \ell) e^{-\ell m} \leq\left(c_{n} / \ell\right) e^{-\ell m},
\end{aligned}
$$

where $c_{n}=\mathbb{E}\left|w_{1}-\theta_{n}\right| \mathbb{E}\left|\zeta_{1}\right|$. If $c_{n} \leq 1$, then (2.3) of Götze and Hipp [14] clearly holds. If $c_{n}>1$, then $c_{n}$ will be bounded above by some constant $c>0$, therefore the right hand side of (7.3) is bounded above by $(c / \ell) e^{-\ell m / c}$. This means that (2.3) of Götze and Hipp [14] still holds but with $\ell / c$ instead of $\ell$. In conclusion, condition (2.3) of Götze and Hipp [14] holds true in our context of Theorem 3.1 .

We now show that (2.5) of Götze and Hipp 14 also holds true in the context of Theorem 3.1, noting first that in what follows $\mathbb{E}(. / \mathcal{F})$ stands for conditional expected value given the $\sigma$-field $\mathcal{F}$. Condition (2.5) of Götze and Hipp in our context corresponds to the following statement: For any $b>0$, there exists $\ell>0$ such that for $|u|>b$ and all $m, n$ such that $1 / b<m<n$, we have

$$
\begin{aligned}
& \mathbb{E} \mid \mathbb{E}\left(\operatorname { e x p } \left(i u \left[\left(w_{n-m}-\theta_{n}\right) X_{n-m}+\cdots+\left(w_{n-1}-\theta_{n}\right) X_{n-1}\right.\right.\right. \\
& \left.\left.\left.\quad+\left(w_{n}-\theta_{n}\right) X_{n}+\cdots+\left(w_{n+m}-\theta_{n}\right) X_{n+m}\right]\right) / \mathcal{D}_{j}, j \neq n\right) \mid \leq e^{-\ell} .
\end{aligned}
$$

To show that (7.4) holds true under the assumptions of our Theorem 3.1, we let $\Psi_{\zeta}$ and $\Psi_{w}$ respectively denote the characteristic functions of $\zeta_{n}$ and $w_{n}$. Observe now that the left hand side of (7.4) is bounded above by

$$
\begin{aligned}
\mathbb{E} \mid & \mathbb{E}\left(\exp \left(i u\left[\zeta_{n} \sum_{k=0}^{m} a_{k}\left(w_{n+k}-\theta_{n}\right)\right]+i u w_{n-1} X_{n-1}\right) / \mathcal{D}_{j}, j \neq n\right) \mid \\
= & \mathbb{E}\left|\mathbb{E}\left(\mathbb{E}\left(\exp \left(i u\left[\zeta_{n} \sum_{k=0}^{m} a_{k}\left(w_{n+k}-\theta_{n}\right)\right]+i u w_{n-1} X_{n-1}\right) / \mathcal{D}_{j, n}, j \neq n\right) / \mathcal{D}_{j}, j \neq n\right)\right| \\
= & \mathbb{E}\left|\mathbb{E}\left(\exp \left(i u\left[\zeta_{n} \sum_{k=0}^{m} a_{k}\left(w_{n+k}-\theta_{n}\right)\right]\right) \mathbb{E}\left(\exp \left(i u w_{n-1} X_{n-1}\right) / \mathcal{D}_{j, n}, j \neq n\right) / \mathcal{D}_{j}, j \neq n\right)\right| \\
= & \mathbb{E}\left|\Psi_{\zeta}\left(u \sum_{k=0}^{m} a_{k}\left(w_{n+k}-\theta_{n}\right)\right) \Psi_{w}\left(u X_{n-1}\right)\right| \\
& \longrightarrow \mathbb{E}\left|\Psi_{w}\left(u X_{1}\right)\right| \mathbb{E}\left|\Psi_{\zeta}\left(u Z_{n}\right)\right| \text { as } m \rightarrow \infty,
\end{aligned}
$$


where

$$
Z_{n}=\sum_{k=0}^{\infty} a_{k}\left(w_{k+1}-\theta_{n}\right) .
$$

The preceding statement is valid in view of the dominated convergence theorem combined with the use of the well known fact that if $X$ and $Y$ are independent, $h(.,$.$) is a bounded$ function and $g(y)=\mathbb{E}(h(X, y))$ then $\mathbb{E}(h(X, Y) \mid Y)=g(Y)$.

Now, if the first part of assumption $(i)$ of Theorem 3.1 holds then, since for all $n \geq 1$ $Z \neq 0$ a.s., for $|u|>b$ for a given $b$, we let $\delta>0$ and $\epsilon>0$ be such that $P\left(\left|u Z_{n}\right|>\epsilon\right)>$ $1 / 2$ and $\left|\Psi_{\zeta}\left(u Z_{n}\right)\right| 1_{\left|u Z_{n}\right|>\epsilon} \leq 1-2 \delta$. Then we have

$$
\begin{aligned}
\mathbb{E}\left|\Psi_{\zeta}\left(u Z_{n}\right)\right| & =\mathbb{E}\left(\left|\Psi_{\zeta}\left(u Z_{n}\right)\right| 1_{\left|u Z_{n}\right|>\epsilon}\right)+\mathbb{E}\left(\left|\Psi_{\zeta}\left(u Z_{n}\right)\right| 1_{\left|u Z_{n}\right| \leq \epsilon}\right) \\
& \leq(1-2 \delta) P\left(\left|u Z_{n}\right|>\epsilon\right)+1-P\left(\left|u Z_{n}\right|>\epsilon\right)=1-2 \delta P\left(\left|u Z_{n}\right|>\epsilon\right)<1-\delta .
\end{aligned}
$$

In summary, for any $b>0$ there exists $\delta>0$ such that for all $|u|>b$, we have $\mathbb{E}\left(\left|\Psi_{\zeta}\left(u Z_{n}\right)\right|\right)<1-\delta$. Therefore for $m$ sufficiently large, for all $|u|>b$, we have

$$
\mathbb{E}\left|\Psi_{\zeta}\left(u \sum_{k=0}^{m} a_{k} w_{k+1}\right)\right| \leq 1-\delta=e^{-\ell},
$$

for some $\ell>0$, which completes the proof of (17.4). We note that in our proof for (7.4), without loss of generality, we can take $b=\ell$ (possibly by having to work with $\zeta_{j} / \ell$ instead of $\zeta_{j}$ ) to be in the same context as in (2.5) of Götze and Hipp [14. This means that (2.5) of Götze and Hipp [14] holds true under the first part of of assumption $(i)$ of Theorem 3.1.

Alternatively, when the second part of the assumption $(i)$ of Theorem 3.1 holds, we show (2.5) of Götze and Hipp [14] continues to hold true. Recalling that in this case the weights satisfy the Cramer condition rather than the innovations, to establish (2.5) of Götze and Hipp [14] in this case, we first note that the left hand side of (7.5), i.e., $\mathbb{E}\left|\Psi_{\zeta}\left(u \sum_{k=0}^{m} a_{k}\left(w_{n+k}-\theta_{n}\right)\right) \Psi_{w}\left(u X_{n-1}\right)\right|$, is bounded above, uniformly in $m \geq 1$, by $E\left|\Psi_{w}\left(u X_{1}\right)\right|$. Since in this case we have $X_{1} \neq 0$ a.s., for given $b$ and for $|u|>b$, we let $\delta>0$ and $\epsilon>0$ be such that $P\left(\left|u X_{1}\right|>\epsilon\right)>1 / 2$ and $\left|\Psi_{w}\left(u X_{1}\right)\right| 1_{\left|u X_{1}\right|>\epsilon} \leq 1-2 \delta$. Then, we have

$$
\begin{aligned}
\mathbb{E}\left|\Psi_{w}\left(u X_{1}\right)\right| & =\mathbb{E}\left(\left|\Psi_{w}\left(u X_{1}\right)\right| 1_{\left|u X_{1}\right|>\epsilon}\right)+\mathbb{E}\left(\left|\Psi_{w}\left(u X_{1}\right)\right| 1_{\left|u X_{1}\right| \leq \epsilon}\right) \\
& \leq(1-2 \delta) P\left(\left|u X_{1}\right|>\epsilon\right)+1-P\left(\left|u X_{1}\right|>\epsilon\right)=1-2 \delta P\left(\left|u X_{1}\right|>\epsilon\right)<1-\delta .
\end{aligned}
$$

The preceding relation implies that, for any $b>0$ there exists $\delta>0$ such that for all $|u|>b$, we have $\mathbb{E}\left(\left|\Psi_{w}\left(u X_{1}\right)\right|\right)<1-\delta$, which implies that (2.5) of Götze and Hipp [14] holds true under the second part of of assumption $(i)$ of Theorem 3.1 .

Finally, in our context, condition (2.6) of Götze and Hipp [14] is clearly satisfied with our choice of $\mathcal{D}_{j}=\sigma\left(w_{j-1}, \zeta_{j}\right)$ in view of the Dynkin's $\pi-\lambda$ theorem (cf., e.g., Billingsley [7]).

Now the proof of Theorem 3.1 is complete.

We note that the preceding proof for Theorem 3.1 implies the validity of (3.1) when $\mathbb{E}\left|\zeta_{1}\right|^{5}<\infty$ and $\mathbb{E}\left|w_{1}\right|^{5}<\infty$, and also that of $\left(\underline{3.3)}\right.$ on assuming $\mathbb{E}\left|\zeta_{1}\right|^{4}<\infty$ and $\mathbb{E}\left|w_{1}\right|^{4}<\infty$, provided that in both cases assumptions $(i)$ and (ii) of Theorem 3.1 hold. 


\section{Proof of Theorem 4.1}

Our arguments below to prove this theorem are valid for both i.i.d. and symmetric multinomial weights as specified in Theorem 4.1. We also note that we only give the proof for the conditional statement (4.1) as it implies the unconditional statement (4.2), in view of the dominated convergence theorem.

For the use in the proof of this theorem, as well as in the proof of Theorem 4.2 we define

$$
\begin{aligned}
K & :=\lim _{n \rightarrow \infty} \mathbb{E}\left(w_{1}-\theta_{n}\right)^{2}, \\
K^{\prime} & :=\lim _{n \rightarrow \infty} \mathbb{E}\left(\left(w_{1}-\theta_{n}\right)\left(w_{2}-\theta_{n}\right)\right) .
\end{aligned}
$$

We note that, by virtue of Corollary $2.1 K$ and $K^{\prime}$ are positive constants and $K^{\prime}<K$.

For the ease of the notation, without loss of generality we assume that $\mu=0$ and also define

$$
\begin{aligned}
\mathfrak{R}_{n, w} & :=\gamma_{0} \sum_{i=1}^{n}\left(w_{i}-\theta_{n}\right)^{2}+2 \sum_{h=1}^{n} \gamma_{h} \sum_{j=1}^{n-h}\left(w_{j}-\theta_{n}\right)\left(w_{j+h}-\theta_{n}\right) \\
& =\operatorname{Var}\left(\sum_{i=1}^{n}\left(w_{i}-\theta_{n}\right) X_{i} \mid w_{1}, \cdots, w_{n}\right),
\end{aligned}
$$

In view of Theorem 2.2 of Abadir et al. (2014), the proof of Theorem 4.1 will result if we show that as $n \rightarrow \infty$,

$$
\begin{gathered}
\mathfrak{R}_{n, w} /\left(n \mathfrak{D}_{n, X, w}\right)-1=o_{P_{w}}(1), \\
\max _{1 \leq i \leq n}\left|w_{i}-\theta_{n}\right| / \sqrt{n \mathfrak{D}_{n, X, w}}=o_{P_{w}}(1),
\end{gathered}
$$

and

$$
\sum_{i=1}^{n}\left(w_{i}-\theta_{n}\right)^{2} /\left(n \mathfrak{D}_{n, X, w}\right)=O_{P_{w}}(1) .
$$

We remark that our condition (17.10) relates to condition (ii) of Theorem 2.2 of Abadir et al. (2014). The latter condition which is intended for constant weights for linear processes reads as follows: $\exists C>0$ such that

$$
\frac{1}{\sigma^{2}} \sup _{n \geq 1} \sum_{j=1}^{n} z_{n j}^{2} \leq C,
$$

where $z_{n j}$ are non-random weights, $\sigma^{2}=\lim _{n \rightarrow \infty} \sum_{i=1}^{n} z_{n j} X_{i}$ and $X_{i}, 1 \leq i \leq n$, are the first $n$ terms of the linear process (2.1). The preceding condition can, conveniently and equivalently, be replaced by

$$
\frac{1}{\sigma^{2}} \sum_{j=1}^{n} z_{n j}^{2}=O(1), \quad \text { as } n \rightarrow \infty .
$$


Hence, in the stochastic context of our Theorem 4.1, the deterministic statement (7.11) is replaced by condition (7.10).

We now establish (7.8) by writing

$$
\begin{aligned}
& \mathfrak{R}_{n, w} /\left(n \mathfrak{D}_{n, X, w}\right)-1 \\
& =\frac{\gamma_{0} \frac{n^{-2 d}}{n} \sum_{i=1}^{n}\left(w_{i}-\theta_{n}\right)^{2}+2 \frac{n^{-2 d}}{n} \sum_{h=1}^{n} \gamma_{h} \sum_{j=1}^{n-h}\left(w_{j}-\theta_{n}\right)\left(w_{j+h}-\theta_{n}\right)}{\gamma_{0} n^{-2 d} \mathbb{E}\left(w_{1}-\theta_{n}\right)^{2}+2 n^{-2 d} \mathbb{E}\left[\left(w_{1}-\theta_{n}\right)\left(w_{2}-\theta_{n}\right)\right] \sum_{h=1}^{n}\left(1-\frac{h}{n}\right) \gamma_{h}}-1 \\
& =\left[\gamma_{0} n^{-2 d}\left(\frac{\sum_{i=1}^{n}\left(w_{i}-\theta_{n}\right)^{2}}{n}-\mathbb{E}\left(w_{1}-\theta_{n}\right)^{2}\right)\right. \\
& \left.+2 n^{-2 d} \sum_{h=1}^{n} \gamma_{h}\left(\frac{1}{n} \sum_{j=1}^{n-h}\left(w_{j}-\theta_{n}\right)\left(w_{j+h}-\theta_{n}\right)-\mathbb{E}\left(\left(w_{1}-\theta_{n}\right)\left(w_{2}-\theta_{n}\right)\right)\right)\right] \times \\
& {\left[\gamma_{0} n^{-2 d} \mathbb{E}\left(w_{1}-\theta_{n}\right)^{2}+2 n^{-2 d} \mathbb{E}\left(\left(w_{1}-\theta_{n}\right)\left(w_{2}-\theta_{n}\right)\right) \sum_{h=1}^{n}\left(1-\frac{h}{n}\right) \gamma_{h}\right]^{-1} .}
\end{aligned}
$$

Considering that, as $n \rightarrow \infty$, for i.i.d. and symmetric multinomial weights we have

$$
\begin{gathered}
\gamma_{0} n^{-2 d} \mathbb{E}\left(w_{1}-\theta_{n}\right)^{2}+2 n^{-2 d} \mathbb{E}\left(\left(w_{1}-\theta_{n}\right)\left(w_{2}-\theta_{n}\right)\right) \sum_{h=1}^{n}\left(1-\frac{h}{n}\right) \gamma_{h} \\
\rightarrow \begin{cases}\gamma_{0}\left(K-K^{\prime}\right)+K^{\prime} s_{X}^{2}>0, & \text { when } d=0, \\
K^{\prime} s_{X}^{2}>0, & \text { when } 0<d<1 / 2,\end{cases}
\end{gathered}
$$

where

$$
s_{X}^{2}:=\lim _{n \rightarrow+\infty} \operatorname{Var}\left(n^{1 / 2-d} \bar{X}_{n}\right)=\lim _{n \rightarrow+\infty} n^{-2 d}\left\{\gamma_{0}+2 \sum_{h=1}^{n-1} \gamma_{h}(1-h / n)\right\} .
$$

From (7.13), the relation (7.8) will follow if we show that, as $n \rightarrow \infty$,

$$
\frac{1}{n} \sum_{i=1}^{n}\left(w_{i}-\theta_{n}\right)^{2}-\mathbb{E}\left(w_{1}-\theta_{n}\right)^{2}=o_{P_{w}}(1)
$$

and

$$
n^{-2 d} \sum_{h=1}^{n} \gamma_{h} \frac{1}{n} \sum_{j=1}^{n-h}\left(\left(w_{j}-\theta_{n}\right)\left(w_{j+h}-\theta_{n}\right)-\mathbb{E}\left(\left(w_{1}-\theta_{n}\right)\left(w_{2}-\theta_{n}\right)\right)\right)=o_{P_{w}}(1) .
$$

When the weights are i.i.d., (7.14) is a consequence of the law of large numbers for row-wise i.i.d. triangular arrays of random variables, in view of the assumption that 
$\mathbb{E}\left|w_{1}\right|^{3}<\infty$, as in scheme (RS) and also in Theorem 4.1.

We now prove that (7.14) continues to hold for the case when $\left(w_{1}, \ldots, w_{n}\right) \stackrel{d}{=}$ Multinomial $(n ; 1 / n, \cdots, 1 / n)$, by writing

$$
\begin{aligned}
& P\left(\left|\frac{1}{n} \sum_{i=1}^{n}\left(\left(w_{i}-\theta_{n}\right)^{2}-\mathbb{E}\left(w_{1}-\theta_{n}\right)^{2}\right)\right|>\epsilon\right) \\
& \leq \epsilon^{-2} \frac{n}{n^{2}} \mathbb{E}\left(\left(w_{1}-\theta_{n}\right)^{2}-\mathbb{E}\left(w_{1}-\theta_{n}\right)^{2}\right)^{2} \\
& +\epsilon^{-2} \frac{n(n-1)}{n^{2}} \mathbb{E}\left[\left(\left(w_{1}-\theta_{n}\right)^{2}-\mathbb{E}\left(w_{1}-\theta_{n}\right)^{2}\right)\left(\left(w_{2}-\theta_{n}\right)^{2}-\mathbb{E}\left(w_{2}-\theta_{n}\right)^{2}\right)\right] .
\end{aligned}
$$

The first expectation above on the right hand side of (7.16) is $O(1)$, as $n \rightarrow \infty$. This conclusion is a consequence of conditions $(i)$ of Theorem 4.1. To show the asymptotic negligibility of the right hand side of (7.16), it remains to show that the second expectation in it is $o(1)$, as $n \rightarrow \infty$. To do so we write,

$$
\begin{aligned}
\mathbb{E} & {\left[\left(\left(w_{1}-\theta_{n}\right)^{2}-\mathbb{E}\left(w_{1}-\theta_{n}\right)^{2}\right)\left(\left(w_{2}-\theta_{n}\right)^{2}-\mathbb{E}\left(w_{2}-\theta_{n}\right)^{2}\right)\right] } \\
= & \mathbb{E}\left(\left(w_{1}-\theta_{n}\right)^{2}\left(w_{2}-\theta_{n}\right)^{2}\right)-\mathbb{E}^{2}\left(w_{1}-\theta_{n}\right)^{2} \\
= & \mathbb{E}\left(\left(w_{1} w_{2}\right)^{2}-2\left(w_{1}\right)^{2} w_{2}+\theta_{n}^{2}\left(w_{1}\right)^{2}-2 \theta_{n} w_{1}\left(w_{2}\right)^{2}+4 \theta_{n}^{2} w_{1} w_{2}-2 \theta_{n}^{3} w_{1}\right. \\
& \left.+\theta_{n}^{2}\left(w_{2}\right)^{2}-2 \theta_{n}^{3} w_{2}+\theta_{n}^{4}\right)-\mathbb{E}^{2}\left(w_{1}\right)^{2}-4 \mathbb{E}\left(w_{1}\right) \theta_{n}^{2}-\theta_{n}^{4}+4 \mathbb{E}^{2}\left(w_{1}\right)^{2} \mathbb{E}\left(w_{1}\right) \theta_{n} \\
& -2 \mathbb{E}^{2}\left(w_{1}\right)+4 \theta_{n}^{3} \mathbb{E}\left(w_{1}\right) \\
\sim & \left(4-4 \theta^{*}+2\left(\theta^{*}\right)^{2}-4 \theta^{*}+4\left(\theta^{*}\right)^{2}-2\left(\theta^{*}\right)^{3}+2\left(\theta^{*}\right)^{2}-2\left(\theta^{*}\right)^{3}+\left(\theta^{*}\right)^{4}\right) \\
& -4-4\left(\theta^{*}\right)^{2}-\left(\theta^{*}\right)^{4}+8 \theta^{*}-4\left(\theta^{*}\right)^{2}+4\left(\theta^{*}\right)^{3} \\
= & 0 .
\end{aligned}
$$

Therefore, (7.14) holds true for symmetric multinomial weights too.

As for (7.15), when weights are i.i.d., for all $\epsilon>0$, we have

$$
\begin{aligned}
& P\left(n^{-2 d}\left|\sum_{h=1}^{n} \gamma_{h} \frac{1}{n} \sum_{j=1}^{n-h}\left(\left(w_{j}-\theta_{n}\right)\left(w_{j+h}-\theta_{n}\right)-\mathbb{E}\left(\left(w_{1}-\theta_{n}\right)\left(w_{2}-\theta_{n}\right)\right)\right)\right|>\epsilon\right) \\
& \leq \epsilon^{-1} n^{-2 d} \sum_{h=1}^{n}\left|\gamma_{h}\right| \mathbb{E}^{1 / 2}\left(\frac{1}{n} \sum_{j=1}^{n-h}\left(\left(w_{j}-\theta_{n}\right)\left(w_{j+h}-\theta_{n}\right)-\mathbb{E}\left(\left(w_{1}-\theta_{n}\right)\left(w_{2}-\theta_{n}\right)\right)\right)\right)^{2} .
\end{aligned}
$$


Now observe that for all $h \geq 1$,

$$
\begin{aligned}
& \mathbb{E}\left(\frac{1}{n} \sum_{j=1}^{n-h}\left(\left(w_{j}-\theta_{n}\right)\left(w_{j+h}-\theta_{n}\right)-\mathbb{E}\left(\left(w_{1}-\theta_{n}\right)\left(w_{2}-\theta_{n}\right)\right)\right)\right)^{2} \\
& =\frac{1}{n^{2}} \sum_{j=1}^{n-h} \mathbb{E}\left(\left(w_{j}-\theta_{n}\right)\left(w_{j+h}-\theta_{n}\right)-\mathbb{E}^{2}\left(w_{1}-\theta_{n}\right)\right)^{2} \\
& =\frac{n-h}{n^{2}}\left(\mathbb{E}\left(\left(w_{1}-\theta_{n}\right)\left(w_{2}-\theta_{n}\right)\right)^{2}-\mathbb{E}^{4}\left(w_{1}-\theta_{n}\right)\right)
\end{aligned}
$$

Substituting the preceding relation into the right hand side of (7.18), in view of conditions (i) and (ii) of this theorem, we conclude that

$$
\begin{aligned}
& \epsilon^{-1} n^{-2 d} \sum_{h=1}^{n}\left|\gamma_{h}\right| \frac{\sqrt{n-h}}{n}\left(\mathbb{E}^{2}\left(\left(w_{1}-\theta_{n}\right)^{2}\right)-\mathbb{E}^{4}\left(w_{1}-\theta_{n}\right)\right)^{1 / 2} \\
& =o(1), \text { as } n \rightarrow \infty .
\end{aligned}
$$

The preceding convergence to zero holds uniformly in $h$ since $\sup _{1 \leq h \leq n-1} \sqrt{n-h} / n=$ $\sqrt{n-1} / n \rightarrow 0$ and $n^{-2 d} \sum_{h=1}^{\infty}|\gamma(h)|$ is bounded. Now the proof of (7.15) in the case of i.i.d. weights is complete.

The proof of (7.15) when the weights are symmetric multinomial also begins with the inequality (7.18). In this case, in view of condition $(i)$, as $n \rightarrow \infty$, we have

$$
\begin{aligned}
& \mathbb{E}\left(\left(w_{1}-\theta_{n}\right)\left(w_{2}-\theta_{n}\right)\right)^{2}-\mathbb{E}^{2}\left(\left(w_{1}-\theta_{n}\right)\left(w_{2}-\theta_{n}\right)\right)=O(1), \\
& \mathbb{E}\left\{\left(\left(w_{1}-\theta_{n}\right)\left(w_{2}-\theta_{n}\right)-\mathbb{E}\left(\left(w_{1}-\theta_{n}\right)\left(w_{2}-\theta_{n}\right)\right)\right)\right. \\
&\left.\quad \times\left(\left(w_{3}-\theta_{n}\right)\left(w_{4}-\theta_{n}\right)-\mathbb{E}\left(\left(w_{3}-\theta_{n}\right)\left(w_{4}-\theta_{n}\right)\right)\right)\right\} \\
& \sim\left\{1-2 \theta^{*}+\left(\theta^{*}\right)^{2}-2 \theta^{*}+4\left(\theta^{*}\right)^{2}-2\left(\theta^{*}\right)^{3}+\left(\theta^{*}\right)^{2}-2\left(\theta^{*}\right)^{3}+\left(\theta^{*}\right)^{4}\right. \\
&\left.\quad-1-4\left(\theta^{*}\right)^{2}-\left(\theta^{*}\right)^{4}+4 \theta^{*}-2\left(\theta^{*}\right)^{2}+4\left(\theta^{*}\right)^{3}\right\} \\
&=0
\end{aligned}
$$

These last two approximations imply that the right hand side of (17.18) is, asymptotically in $n$ and uniformly in $h$, negligible which means that (7.15) holds for symmetric multinomial weights. This completes the proof of (7.8).

We now give a unified argument for both i.i.d. and symmetric multinomial weights to 
prove (7.9). The proof is also valid for both short and long memory data and it begins with observing that, as $n \rightarrow \infty$, we have

$$
\frac{1}{\sqrt{n}} \max _{1 \leq j \leq n}\left|w_{j}-\theta_{n}\right|=o_{P_{w}}(1)
$$

Considering that $\theta_{n}$ is bounded, the weight $w_{j}$, for each $n$, are identically distributed and, since, in the scheme (RS) the weights are so that $\sup _{n \geq 1} \mathbb{E}\left|w_{1}\right|^{3}<\infty$, the proof of (7.21) follows from the following argument.

$$
\begin{aligned}
P\left(\max _{1 \leq j \leq n}\left(w_{j}\right)^{2}>n \epsilon\right) & \leq n P\left(\left(w_{1}\right)^{2}>n \epsilon\right) \\
& \leq \epsilon^{-3 / 2} n^{-1 / 2} \mathbb{E}\left|w_{1}\right|^{3} \leq \epsilon^{-3 / 2} n^{-1 / 2} \mathbb{E}\left|w_{1}\right|^{3} \rightarrow 0
\end{aligned}
$$

In view of the conditions $(i)$ and $(i i)$ of Theorem 4.1, for some finite number $A$, we have

$$
\begin{aligned}
& \mathbb{E}\left(w_{1}-\theta_{n}\right)^{2} \gamma_{0}+2 \mathbb{E}\left(\left(w_{1}-\theta_{n}\right)\left(w_{2}-\theta_{n}\right)\right) \sum_{h=1}^{n}\left(1-\frac{h}{n}\right) \gamma_{h} \\
& \longrightarrow K \gamma_{0}+2 K^{\prime} \sum_{h=1}^{\infty} \gamma_{h}= \begin{cases}A, & \text { when } X_{j} \text { of short memory } \\
\infty, & \text { when } X_{j} \text { of long memory }\end{cases}
\end{aligned}
$$

Recall that $K^{\prime}<K$. This, in turn, implies that $A>0$. This together with (7.21) completes the proof of (7.9).

To complete the proof of Theorem 4.1, we need to prove (7.10). The validity of (7.10), for both i.i.d. and symmetric multinomial weights, by virtue of (7.14) and conditions (ii) of Theorem 4.1, results from the following weak law of large numbers, as $n \rightarrow \infty$.

$$
\begin{aligned}
& \frac{(1 / n) \sum_{j=1}^{n}\left(w_{j}-\theta_{n}\right)^{2}}{\mathbb{E}\left(w_{1}-\theta_{n}\right)^{2} \gamma_{0}+2 \mathbb{E}\left(\left(w_{1}-\theta_{n}\right)\left(w_{2}-\theta_{n}\right)\right) \sum_{h=1}^{n}\left(1-\frac{h}{n}\right) \gamma_{h}} \\
& \stackrel{K}{\longrightarrow} \frac{P_{w}}{\gamma_{0} K+2 K^{\prime} \sum_{h=1}^{\infty} \gamma_{h}}= \begin{cases}A^{\prime}>0, & \text { when } X_{j} \text { of short memory, } \\
0, & \text { when } X_{j} \text { of long memory }\end{cases}
\end{aligned}
$$

This completes the proof of (17.10) as well as that of Theorem 4.1.

\section{Proof of Theorem 4.2}

In the proof of this theorem, as well as in the proof of Theorem 6.1, for the ease of the notation we let $P_{X \mid w}($.$) stand for the conditional probability P\left(. \mid w_{1}, \cdots, w_{n}\right)$.

As $n, q \rightarrow \infty$, in such a way that $q=O\left(n^{1 / 2}\right)$, under the conditions of this theorem, 
from the approximation (2.10) of Theorem 2.1 of Abadir et al. [2, that holds true for $d$ and $\hat{d}$, we have

$$
q^{-2 d} \bar{\gamma}_{0}+2 q^{-2 d} \sum_{h=1}^{q} \bar{\gamma}_{h}\left(1-\frac{h}{q}\right) \longrightarrow s_{X}^{2},
$$

where $s_{X}^{2}$ is as defined right after (7.13). This, in turn, implies that, as $n, q \rightarrow \infty$, in such a way that $q=O\left(n^{1 / 2}\right)$, we also have

$$
\begin{aligned}
& q^{-2 d} \mathcal{S}_{n, q, w}=q^{-2 d} \mathbb{E}\left(w_{1}-\theta_{n}\right)^{2} \bar{\gamma}_{0}+2 q^{-2 d} \mathbb{E}\left(\left(w_{1}-\theta_{n}\right)\left(w_{2}-\theta_{n}\right)\right) \sum_{h=1}^{q} \bar{\gamma}_{h}\left(1-\frac{h}{q}\right) \\
& \stackrel{P}{\longrightarrow} \begin{cases}\gamma_{0}\left(K-K^{\prime}\right)+K^{\prime} s_{X}^{2}>0, & \text { when } d=0, \\
K^{\prime} s_{X}^{2}>0, & \text { when } 0<d<1 / 2,\end{cases}
\end{aligned}
$$

where the constant $K$ is as in (7.6) and $K^{\prime}$ is as in (7.7). The preceding convergence means that the Studentizing sequence $q^{-2 d} \mathcal{S}_{n, q, w}$ asymptotically coincides with the limit of $n \mathcal{D}_{n, X, w}$, which is the normalizing sequence for $T_{n, X, w}\left(\theta_{n}\right)$, as in (2.6). In Theorem 4.1 we showed $T_{n, X, w}\left(\theta_{n}\right)$ has standard normal limiting distribution. Considering that

$$
G_{n, X, w}\left(\theta_{n}, d\right)=T_{n, X, w}\left(\theta_{n}\right) \sqrt{\left(n \mathcal{D}_{n, X, w}\right) /\left(q^{-2 d} \mathcal{S}_{n, q, w}\right)},
$$

we conclude, from Slutsky theorem, that $G_{n, X, w}\left(\theta_{n}, d\right)$ also convergence to standard normal. The relations (7.22) and (7.23) are also true when the memory parameter $d$ is replaced by its estimator $\hat{d}$, provided that $\hat{d}-d=o_{p}(1 / \log n)$.

Now the proof of Theorem 4.2 is complete.

\section{Proof of Theorem 6.1}

The proof of Theorem 6.1, for both i.i.d. and symmetric multinomial weights, will follow if we show that

$$
\begin{aligned}
& P_{X \mid w}\left\{\left|\left(\left(q^{-2 d} \hat{\mathcal{S}}_{n, q, w}\right) /\left(q^{-2 d} \mathcal{S}_{n, q, w}\right)\right)-1\right|>\epsilon\right\} \\
= & P_{X \mid w}\left\{\left|\frac{q^{-2 d} \bar{\gamma}_{0} \frac{1}{n} \sum_{j=1}^{n}\left(w_{j}-\theta_{n}\right)^{2}+2 q^{-1-2 d} \sum_{h=1}^{q} \bar{\gamma}_{h} \sum_{j=1}^{q-h}\left(w_{j}-\theta_{n}\right)\left(w_{j+h}-\theta_{n}\right)}{q^{-2 d} \bar{\gamma}_{0} \mathbb{E}\left(w_{1}-\theta_{n}\right)^{2}+2 q^{-2 d} \mathbb{E}\left(\left(w_{1}-\theta_{n}\right)\left(w_{2}-\theta_{n}\right)\right) \sum_{h=1}^{q} \bar{\gamma}_{h}\left(1-\frac{h}{q}\right)}-1\right|>\epsilon\right\} \\
= & o_{P_{w}}(1), \quad \text { as } n \rightarrow \infty,
\end{aligned}
$$

where $\epsilon$ is an arbitrary positive constant. By virtue of (7.22), the proof of (7.24), as $n, q \rightarrow \infty$ such that $q=O\left(n^{1 / 2}\right)$, will follow from (7.14) combined with

$$
P_{X \mid w}\left(\left|q^{-2 d} \sum_{h=1}^{q} \bar{\gamma}_{h} B_{n, q, w}(h)\right|>\epsilon\right)=o_{P_{w}}(1),
$$


where

$$
B_{n, q, w}(h):=q^{-1} \sum_{j=1}^{q-h}\left(\left(w_{j}-\theta_{n}\right)\left(w_{j+h}-\theta_{n}\right)-\mathbb{E}\left(\left(w_{1}-\theta_{n}\right)\left(w_{2}-\theta_{n}\right)\right)\right) .
$$

The proof of (7.25) is a modification of the proof of (6.14) in Csörgö et al. [10].

For convenient reference here we give the proof of (7.25) for both i.i.d. and symmetric multinomial weights. To do so, without loss of generality, we assume that $\mu=\mathbb{E} X_{1}=0$ and, for each $1 \leq h \leq q$, we define

$$
\gamma_{h}^{*}:=\frac{1}{n} \sum_{i=1}^{n-h} X_{i} X_{i+h} .
$$

Observe that, for $\varepsilon_{1}, \varepsilon_{2}>0$, we have

$$
\begin{aligned}
& P\left\{P_{X \mid w}\left(q^{-2 d}\left|\sum_{h=1}^{q} \bar{\gamma}_{h} B_{n, q, w}(h)\right|>2 \varepsilon_{1}\right)>\varepsilon_{2}\right\} \\
& \leq P\left\{P_{X \mid w}\left(q^{-2 d}\left|\sum_{h=1}^{q}\left(\bar{\gamma}_{h}-\gamma_{h}^{*}\right) B_{n, q, w}(h)\right|>\varepsilon_{1}\right)>\varepsilon_{2}\right\} \\
& \quad+P\left\{P_{X \mid w}\left(q^{-2 d}\left|\sum_{h=1}^{q} \gamma_{h}^{*} B_{n, q, w}(h)\right|>\varepsilon_{1}\right)>\varepsilon_{2}\right\} .
\end{aligned}
$$

We now show that the first term in (7.27) is asymptotically negligible, noting first that

$$
\begin{aligned}
\sum_{h=1}^{q}\left(\bar{\gamma}_{h}-\gamma_{h}^{*}\right) B_{n, q, w}(h)= & -\frac{\bar{X}_{n}}{n} \sum_{h=1}^{q} B_{n, q, w}(h) \sum_{i=1}^{n-h} X_{i}-\frac{\bar{X}_{n}}{n} \sum_{h=1}^{q} B_{n, q, w}(h) \sum_{i=1}^{n-h} X_{i+h} \\
& +\bar{X}^{2} \sum_{h=1}^{q} B_{n, q, w}(h) \\
\sim & -\bar{X}^{2} \sum_{h=1}^{q} B_{n, q, w}(h) \text { uniformly in } h \text { in probability }-P_{X \mid w}
\end{aligned}
$$

where, in the preceding conclusion, generically, $Y_{n} \sim Z_{n}$ in probability- $P$ means $Y_{n}=$ $Z_{n}\left(1+o_{P}(1)\right)$. The approximation in (7.28) is true since, for $\varepsilon>0$ we have

$$
\begin{aligned}
P\left(\cup_{1 \leq h \leq q}\left|\bar{X}_{n}-\frac{\sum_{i=1}^{n-h} X_{i}}{n}\right|>\varepsilon\right) & \leq q P\left(\left|\frac{\sum_{i=n-h+1}^{n} X_{i}}{n}\right|>\varepsilon\right) \\
& \leq \varepsilon^{-4} q \frac{(h-1)^{4}}{n^{4}} \mathbb{E}\left(X_{1}^{4}\right) \\
& \leq \varepsilon^{-4} \frac{q^{5}}{n^{4}} \mathbb{E}\left(X_{1}^{4}\right) \rightarrow 0 \text {, as } n \rightarrow \infty .
\end{aligned}
$$


The preceding is true since $1 \leq h \leq q$ and $q=O\left(n^{1 / 2}\right)$, as $n, q \rightarrow \infty$.

We note that for $0 \leq d<1 / 2$, as $n \rightarrow \infty$, we have that $n^{1 / 2-d} \bar{X}_{n}=O_{P}(1)$. The latter conclusion, in view of the equivalence in (17.28), implies that, for each $\varepsilon_{1}, \varepsilon_{2}>0$, there exists $\varepsilon>0$ such that

$$
\begin{aligned}
& P\left\{P_{X \mid w}\left(q^{-2 d}\left|\sum_{h=1}^{q}\left(\bar{\gamma}_{h}-\gamma_{h}^{*}\right) B_{n, q, w}(h)\right|>\varepsilon_{1}\right)>\varepsilon_{2}\right\} \\
\sim & P\left\{\frac{q^{-2 d}}{n^{1-2 d}} \sum_{h=1}^{q}\left|B_{n, q, w}(h)\right|>\varepsilon\right\} \\
\leq & \varepsilon^{-1} \frac{q^{-2 d}}{n^{1-2 d}} \sum_{h=1}^{q} \mathbb{E}\left(\left|B_{n, q, w}(h)\right|\right) .
\end{aligned}
$$

From condition $(\mathrm{ii})$ of Theorem 4.1, that is also assumed in Theorem 4.2, there exists a constant $\mathcal{L}$ whose value does not depend on $n$ such that $\sup _{n \geq 2} \sup _{1 \leq h \leq q} \mathbb{E}\left(\left|B_{n, q, w}(h)\right|\right)<$ $\mathcal{L}$. Hence, (7.29) can be bounded above by

$$
\mathcal{L} \varepsilon^{-1} \frac{q^{1-2 d}}{n^{1-2 d}} \longrightarrow 0
$$

as $n, q \rightarrow \infty$ in such away that $q=O\left(n^{1 / 2}\right)$. This means that the first term in (7.27) is asymptotically negligible. To establish (7.24) we show that the second term in (7.27) is also asymptotically negligible. To prove this negligibility, we first define

$$
\gamma_{h}^{* *}:=\frac{1}{n} \sum_{i=1}^{n} X_{i} X_{i+h} .
$$

Now, observe that

$$
\begin{aligned}
P\left\{\cup_{1 \leq h \leq q}\left|\gamma_{h}^{* *}-\gamma_{h}^{*}\right|>\varepsilon\right\} & \leq q P\left\{\frac{1}{n}\left|\sum_{i=n-h+1}^{n} X_{i} X_{i+h}\right|>\varepsilon\right\} \\
& \leq \varepsilon^{-2} \frac{q^{3}}{n^{2}} \mathbb{E}\left(X_{1}^{4}\right) \rightarrow 0
\end{aligned}
$$

as $n, q \rightarrow \infty$ such that $q=O\left(n^{1 / 2}\right)$, hence, as $n, q \rightarrow \infty$ such that $q=O\left(n^{1 / 2}\right)$, using an argument similar to those used for (17.27) and (7.29), with $\gamma_{h}^{*}$ replacing $\bar{\gamma}_{h}$ and $\gamma_{h}^{* *}$ replacing $\gamma_{h}^{*}$ therein, we arrive at

$$
\begin{gathered}
P\left\{P_{X \mid w}\left(q^{-2 d}\left|\sum_{h=1}^{q} \gamma_{h}^{*} B_{n, q, w}(h)\right|>\varepsilon_{1}\right)>\varepsilon_{2}\right\} \\
\sim P\left\{P_{X \mid w}\left(q^{-2 d}\left|\sum_{h=1}^{q} \gamma_{h}^{* *} B_{n, q, w}(h)\right|>\varepsilon_{1}\right)>\varepsilon_{2}\right\} .
\end{gathered}
$$


Therefore, in order to prove (7.24), it suffices to show that, as $n, q \rightarrow \infty$ so that $q=$ $O\left(n^{1 / 2}\right)$,

$$
P\left\{P_{X \mid w}\left(q^{-2 d}\left|\sum_{h=1}^{q} \gamma_{h}^{* *} B_{n, q, w}(h)\right|>\varepsilon_{1}\right)>\varepsilon_{2}\right\} \rightarrow 0,
$$

The preceding relation, in turn, follows from the following two conclusions: as $n, q \rightarrow \infty$ so that $q=O\left(n^{1 / 2}\right)$,

$$
\sup _{1 \leq h, h^{\prime} \leq q} \mathbb{E}\left(\left|B_{n, q, w}(h) B_{n, q}\left(h^{\prime}\right)\right|\right)=o(1)
$$

and

$$
q^{-4 d} \sum_{h=1}^{q} \sum_{h^{\prime}=1}^{q}\left|\mathbb{E}\left(\gamma_{h}^{* *} \gamma_{h^{\prime}}^{* *}\right)\right|=O(1) .
$$

To prove (7.31), using the Cauchy inequality we write

$$
\begin{aligned}
& \mathbb{E}\left(\left|B_{n, q, w}(h) B_{n, q, w}\left(h^{\prime}\right)\right|\right) \\
\leq & \mathbb{E}\left(B_{n, q, w}(h)\right)^{2} \\
\leq & \frac{q-h}{q^{2}} \mathbb{E}\left(\left(w_{1}-\theta_{n}\right)\left(w_{2}-\theta_{n}\right)-\mathbb{E}\left(\left(w_{1}-\theta_{n}\right)\left(w_{2}-\theta_{n}\right)\right)\right)^{2} \\
+ & \frac{(q-h)(q-h-1)}{q^{2}} \mathbb{E}\left\{\left(\left(w_{1}-\theta_{n}\right)\left(w_{2}-\theta_{n}\right)-\mathbb{E}\left(\left(w_{1}-\theta_{n}\right)\left(w_{2}-\theta_{n}\right)\right)\right)\right. \\
& \left.\times\left(\left(w_{3}-\theta_{n}\right)\left(w_{4}-\theta_{n}\right)-\mathbb{E}\left(\left(w_{3}-\theta_{n}\right)\left(w_{4}-\theta_{n}\right)\right)\right)\right\} .
\end{aligned}
$$

In case of i.i.d. weights, (7.33) is bounded above by $q^{-1} \mathbb{E}^{2}\left(w_{1}-\theta_{n}\right)^{2}$ that vanishes as $q, n \rightarrow \infty$. In case of symmetric multinomial weights, from (17.19) and (7.20) we can see that (7.33) has an upper bound of the form $2 K_{n} / q+k_{n}$, where $K_{n}=O(1)$ and $k_{n}=o(1)$, that also vanishes as $n, q \rightarrow \infty$. The latter conclusion completes the proof of (7.33) and that of (7.31).

In order to establish (7.32), we define

$$
H:=\lim _{s \rightarrow \infty} s^{-2 d} \sum_{\ell=-s}^{s}\left|\gamma_{\ell}\right|
$$

Observe that $H<\infty$. We now carry on with the proof of (7.32), using a generalization of an argument used in the proof of Proposition 7.3.1 of Brockwell and Davis [8] as follows: 


$$
\begin{aligned}
& q^{-4 d} \sum_{h=1}^{q} \sum_{h^{\prime}=1}^{q}\left|\mathbb{E}\left(\gamma_{h}^{* *} \gamma_{h^{\prime}}^{* *}\right)\right| \leq q^{-2 d} \sum_{h=1}^{q}\left|\gamma_{h}\right| q^{-2 d} \sum_{h^{\prime}=1}^{q}\left|\gamma_{h^{\prime}}\right| \\
& +\left(\frac{q}{n}\right)^{1-2 d} n^{-2 d} \sum_{k=-n}^{n}\left|\gamma_{h}\right| q^{-2 d} \sum_{L=-q}^{q}\left|\gamma_{k+L}\right| \\
& +\frac{1}{n} \sum_{k=-n}^{n} q^{-2 d} \sum_{h^{\prime}=1}^{q}\left|\gamma_{k+h^{\prime}}\right| q^{-2 d} \sum_{h=1}^{q}\left|\gamma_{k-h}\right| \\
& +\frac{q^{-2 d}}{n^{1-2 d}} n^{-2 d} \sum_{i=1}^{n} \sum_{k=-n}^{n}\left|a_{i} a_{i+k}\right| q^{-d} \sum_{h=1}^{q}\left|a_{i+h}\right| q^{-d} \sum_{h^{\prime}=1}^{q} \mid a_{i+k}\left(\mathrm{Z}_{k} \mathrm{q}_{4} \text {. }\right)
\end{aligned}
$$

It is easy to see that, as $n \rightarrow \infty$, and consequently $q \rightarrow \infty$, the right hand side of the inequality (7.34) converges to the finite limit $3 H^{2}$. Now the proof of $(7.32)$ and also that of Theorem 6.1 are complete.

\section{References}

[1] Abadir, K. M., Distaso, W., Giraitis, L., Koul, H. L. (2014). Asymptoti normality for weighted sums of linear processes. Econometric Theory 30, 252-284.

[2] Abadir, K. M., Distaso, W., Giraitis, L. (2009). Two estimators of the long-run variance: beyond short memory. Journal of Econometrics 150, 56-70.

[3] Andrews, D. W. K. (1991). Heteroskedasticity and autocorrelation consistent covariance matrix estimation. Econometrica 59, 817-858.

[4] Barndorff-Nielsen, O. E., Cox D. R. (1989). Asymptotic techniques for use in statistics. London; New York: Chapman and Hall.

[5] Bhansali, R. J., Kokoszka, P. S. (2001). Estimation of the long-memory parameter: a review of recent developments and an extension. In Proceedings of the Symposium on Inference for Stochastic Processes, I.V. Basawa, C. C. Heyde and R.L. Taylor, eds. IMS Lecture Notes, 125-150.

[6] Bhattacharya, R. N., Rao, R. R. (1976). Normal approximation and asymptotic expansions. New York London Sydney Toronto: John Wiley \& Sons.

[7] Billingsley, P. (2012). Probability and measure. Wiley, 3rd edition, New York.

[8] Brockwell, P. J., Davis R. A. (2009). Time series: theory and methods. Springer Second Editon.

[9] Csörgő, M., Nasari, M. M. (2015). Inference from small and big data sets with error rates. Electronic Journal of Statistics 9, 535-566. 
[10] Csörgö, M., Nasari, M. M., Ould-Haye, M. (2017). Randomized pivots for means of short and long memory linear processes. Bernoulli 23, 2558-2586.

[11] Efron, B. (1979). Another look at the Jackknife. Annals of Statistics 7, 1-26.

[12] Efron, B. and Tibshirani, R. (1993). An introduction to the bootstrap. Chapman \& Hall.

[13] Eriksson, M. (2008). A simulation method for skewness correction. U.U.D.M. Project Report 2008:22, Uppsala University.

[14] Götze, F., Hipp, C. (1983). Asymptotic expansions for sums of weakly dependent random vectors. Z. Wahrsch. Verw. Gebiete 64, 211-239.

[15] Götze, F., Künsch, H. R. (1996). Second-order correctness of the blockwise bootstrap for stationary observations. The Annals of Statistics 5 1914-1933.

[16] Hall, P. (1986). On the number of bootstrap simulations required to construct a confidence interval. Annals of Statistics 14, 1453-1462.

[17] Hall, P. (1983). Inverting an Edgeworth expansion. Annals of Statistics 11, 569-576.

[18] Hasslet, J., Raftery, A. E. (1989). Space-time modelling with longmemory dependence: assessing Ireland's wind power resource. Applied Statistics 38, 1-50.

[19] Kim, Y. M., Nordman, D. J. (2011). Properties of a block bootstrap under longrange dependence. The Indian Journal of Statistics 73-A, 79-109.

[20] Künsch, H. R. (1989). The jackknife and the bootstrap for general stationary observations. The Annals of Statistics 3, 1217-1241.

[21] Künsch, H. R. (1987). Statistical aspect of self-similar processes. In Y. A. Prohorov \& V. V. Sazonov (Eds.) Procedings of the 1st World Congress of the Bernoulli Society 1, 67-74. VNU Science Press.

[22] Lahiri, S. N. (2003). Resampling methods for dependent data. Springer-Verlag New York, Inc.

[23] Lahiri, S. N. (1993). Refinements in asymptotic expansions for sums of weakly dependent random vectors. Annals of Probability 21, 791-799.

[24] Moulines, E., Soulier, Ph. (2003). Semiparametric spectral estimation for fractional processes: In Theory and applications of long-range dependence. In Dukhan, P., Oppenheim, G. and Taqqu, M. (Eds.) Theory an applications of long-range dependendence. Birkhäuer Boston.

[25] Nasari, M. M. (2016). Pivotal quantities with arbitrary small skewness. arXiv:1605.05985 
[26] Poskitt D. S. (2008). Properties of the sieve bootstrap for fractionally integrated and non-invertible processes. Journal of Time Series Analysis 29, 224-250.

[27] Poskitt, D. Simone, G. Martin, G. (2015) Higher-order improvements of the sieve bootstrap for fractionally integrated processes. Journal of Econometrics 188, 94-110.

[28] Robinson, P. M. (2005). Robust covariance matrix estimation: HAC estimates with long memory/antipersistence correction. Econometric theory 21, 171-180.

[29] Robinson, P. M. (1995). Guassian semiparametric estimation of long range dependence. Annals of Statistics 23, 1630-1661.5)

[30] Senatov, V. V. (1998). Normal approximation: new results, methods and problems. Utrecht, The Netherlands Tokyo, Japan: VSP.

[31] Shorack, G. R. (2000). Probability for statisticians. Springer.

[32] Yatracos, Y. (2002). Assessing the quality of bootstrap samples and of the bootstrap estimates obtained with finite resampling. Statistics and Probability Letters 59, 281292. 